\title{
The Transit Light Source Effect: False Spectral Features and Incorrect Densities for M-dwarf Transiting Planets
}

\author{
Benjamin V. Rackham ${ }^{1,4,5}$ (10), Dániel Apai ${ }^{1,2,5}$ (D), and Mark S. Giampapa ${ }^{3}$ (1D \\ ${ }^{1}$ Department of Astronomy/Steward Observatory, The University of Arizona, 933 N. Cherry Avenue, Tucson, AZ 85721, USA; brackham@as.arizona.edu \\ ${ }^{2}$ Department of Planetary Sciences, The University of Arizona, 1629 E. University Boulevard Tucson, AZ 85721, USA \\ ${ }^{3}$ National Solar Observatory, 950 N. Cherry Avenue, Tucson, AZ 85719, USA \\ Received 2017 October 4; revised 2017 December 5; accepted 2017 December 6; published 2018 January 30
}

\begin{abstract}
Transmission spectra are differential measurements that utilize stellar illumination to probe transiting exoplanet atmospheres. Any spectral difference between the illuminating light source and the disk-integrated stellar spectrum due to starspots and faculae will be imprinted in the observed transmission spectrum. However, few constraints exist for the extent of photospheric heterogeneities in M dwarfs. Here we model spot and faculae covering fractions consistent with observed photometric variabilities for $\mathrm{M}$ dwarfs and the associated $0.3-5.5 \mu \mathrm{m}$ stellar contamination spectra. We find that large ranges of spot and faculae covering fractions are consistent with observations and corrections assuming a linear relation between variability amplitude, and covering fractions generally underestimate the stellar contamination. Using realistic estimates for spot and faculae covering fractions, we find that stellar contamination can be more than $10 \times$ larger than the transit depth changes expected for atmospheric features in rocky exoplanets. We also find that stellar spectral contamination can lead to systematic errors in radius and therefore the derived density of small planets. In the case of the TRAPPIST-1 system, we show that TRAPPIST-1's rotational variability is consistent with spot covering fractions $f_{\text {spot }}=8_{-7}^{+18} \%$ and faculae covering fractions $f_{\text {fac }}=54_{-46}^{+16} \%$. The associated stellar contamination signals alter the transit depths of the TRAPPIST-1 planets at wavelengths of interest for planetary atmospheric species by roughly $1-15 \times$ the strength of planetary features, significantly complicating JWST follow-up observations of this system. Similarly, we find that stellar contamination can lead to underestimates of the bulk densities of the TRAPPIST-1 planets of $\Delta(\rho)=-8_{-20}^{+7} \%$, thus leading to overestimates of their volatile contents.
\end{abstract}

Key words: methods: numerical - planets and satellites: atmospheres - planets and satellites: fundamental parameters - stars: activity - starspots - techniques: spectroscopic

\section{Introduction}

Transmission spectroscopy, the multiwavelength study of transits that reveals the apparent size of the exoplanet as a function of wavelength (e.g., Seager \& Sasselov 2000; Brown 2001), provides the best opportunity to study the atmospheres of small and cool exoplanets in the coming decades. During a transit, exoplanets appear larger at some wavelengths due to absorption or scattering of starlight by their atmospheres. The scale of the signal depends inversely on the square of the stellar radius (Miller-Ricci et al. 2009), prompting a focus on studying exoplanets around M-dwarf stars.

A rapidly growing number of exciting M-dwarf exoplanet systems hosting super-Earth and Earth-mass planets have been discovered to date, including GJ 1132b (Berta-Thompson et al. 2015), LHS 1140b (Dittmann et al. 2017), and the TRAPPIST-1 system (Gillon et al. 2016, 2017; Luger et al. 2017), an ultracool dwarf only 12 pc away hosting a system of seven transiting Earth-sized planets. The low densities of the TRAPPIST-1 planets may indicate high volatile contents, and as many as three of them may have surface temperatures temperate enough for long-lived liquid water to exist (Gillon et al. 2017). Frequent flaring (Vida et al. 2017) and strong XUV radiation from the host star (Wheatley et al. 2017), however, can lead to significant water loss for

\footnotetext{
${ }^{4}$ National Science Foundation Graduate Research Fellow.

5 Earths in Other Solar Systems Team, NASA Nexus for Exoplanet System Science.
}

these planets (Bolmont et al. 2017), and 3D climate modeling suggests that TRAPPIST-1e provides the best opportunity for present-day surface water and an Earth-like temperature in the system (Wolf 2017). While M-dwarf exoplanets provide an excellent opportunity to study small and cool exoplanets (Barstow \& Irwin 2016), they also represent a significant challenge. Spots with covering fractions as low as $1 \%$ on $\mathrm{M}$ dwarfs introduce radial velocity jitter that can mask the presence of habitable-zone Earth-sized exoplanets (Andersen \& Korhonen 2015). Variability monitoring suggests that $1 \%$ $3 \%$ of $\mathrm{M}$ dwarfs have spot covering fractions of $10 \%$ or more (Goulding et al. 2012). In addition to radial velocity jitter, unocculted spots also introduce errors in wavelength-dependent planetary radii recovered from transit observations (e.g., Pont et al. 2008). Given the dependence of density calculations on measurements of exoplanet radii $\left(\rho \propto R^{-3}\right)$, any errors in radius determination are amplified by a factor of 3 in the estimate of the exoplanet bulk density and can lead to significant consequences for the development of accurate exoplanet models.

Unocculted spots are one manifestation of a generic issue with transit observations that we term the "transit light source effect" (Figure 1): any transmission spectroscopic measurement relies on measuring the difference between the incident and transmitted light to identify the absorbers present in the media studied (e.g., Seager \& Sasselov 2000). The level of accuracy with which the incident spectrum is known will directly determine the level of accuracy with which the transmitted light 


\section{The Transit Light Source Effect}

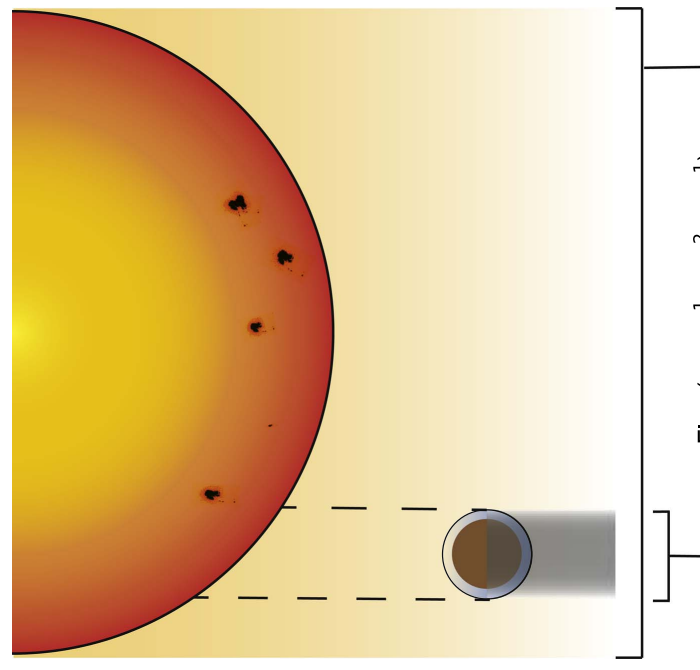

Pre-transit Stellar Disk is the Assumed Light Source

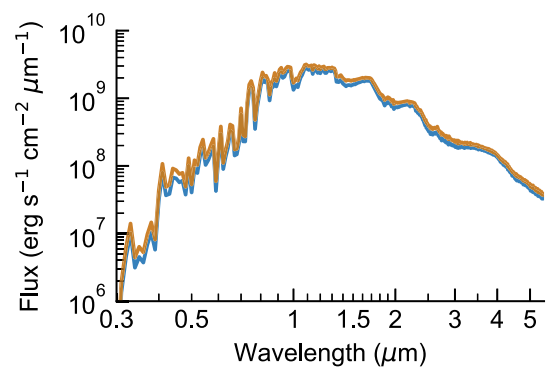

Actual Light Source is the Chord Defined by the Planet's Projection

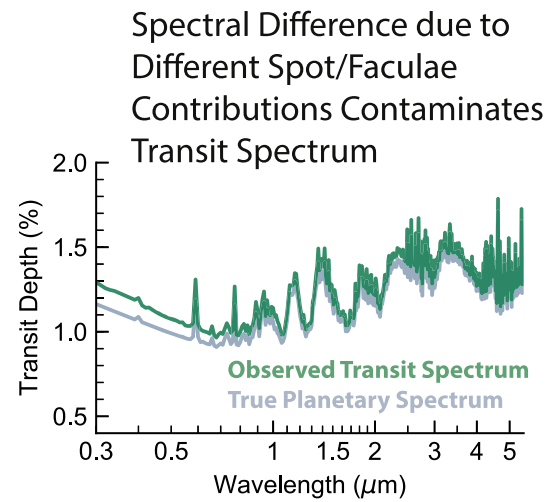

Spectral Difference due to Different Spot/Faculae Contributions Contaminates .

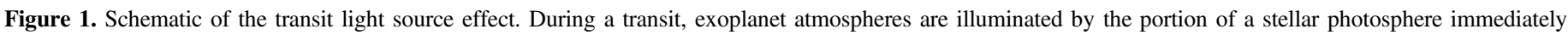

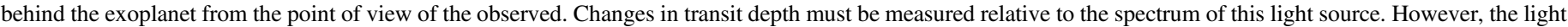

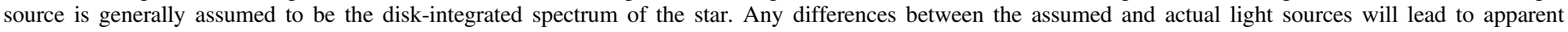
variations in transit depth.

is understood. In the transiting exoplanet case, the incident light is measured by observing the disk-integrated stellar spectrum before the transit (e.g., Brown 2001), the assumption being that the disk-integrated spectrum is identical to the light incident on the planetary atmosphere. However, this is only an approximation: the planet is not occulting the entire stellar disk but only a small region within the transit chord at a given time. Thus, the light source for the transmission measurement is a small time-varying annulus within the stellar disk defined by the planet's projection, the spectrum of which may differ significantly from the disk-averaged spectrum. Such differences are expected due to the fact that stellar atmospheres are rarely perfectly homogeneous, as illustrated by spatially resolved observations of the Sun (e.g., Llama \& Shkolnik 2015, 2016). Cool stellar spots (umbra and penumbra), hot faculae, and even latitudinal temperature gradients will result in a spectral mismatch, even if some of these will not be evident in broadband photometric light curves.

The Sun displays a clear latitudinal dependence of active regions that gives rise to the so-called butterfly diagram (Maunder 1922; Babcock 1961; Mandal et al. 2017). Transiting exoplanets have been proposed to be tools to probe the latitudinal and temporal distributions of active regions in other stars (Dittmann et al. 2009; Llama et al. 2012). High-resolution transit observations can be used to spatially resolve the emergent stellar spectrum along the transit path of the planet (Cauley et al. 2017; Dravins et al. 2017a, 2017b). Morris et al. (2017) recently utilized the highly misaligned exoplanet HAT$\mathrm{P}-11 \mathrm{~b}$ to probe the starspot radii and latitudinal distribution of its K4 dwarf host star and found that, much like the Sun, spots on HAT-P-11 emerge preferentially at two low latitudes. In general, however, orbital planes of transiting exoplanets tend to be more aligned with stellar rotation axes than that of HAT-P$11 \mathrm{~b}$, displaying obliquities of $\lesssim 20^{\circ}$ (Winn et al. 2017). To complicate matters further, unlike the Sun and HAT-P-11, M dwarfs may exhibit spots at all latitudes (Barnes et al. 2001). Thus, stellar latitudes sampled by transit chords may not provide a representative picture of photospheric active regions.
Correcting transmission spectra for photospheric heterogeneities within the transit chord, such as spots (e.g., Pont et al. 2013; Llama \& Shkolnik 2015) and faculae (Oshagh et al. 2014), is possible, provided they are large enough to produce an observable change in the light curve during the transit. Modulations in the shape of the transit light curve can be used to constrain the temperature (Sing et al. 2011) and size (Béky et al. 2014) of the occulted photospheric feature, which determine its contribution to the transmission spectrum, or more simply, time points including the crossing event may be excluded from the transit fit (e.g., Pont et al. 2008; Carter et al. 2011; Narita et al. 2013).

Unocculted heterogeneities, however, represent a more pathological manifestation of the transit light source effect because they do not produce temporal changes in the observed light curve. Previous attempts to correct for unocculted photospheric features have largely relied on photometric monitoring of the exoplanet host star to ascertain the extent of photospheric heterogeneities present (Pont et al. 2008, 2013; Berta et al. 2011; Désert et al. 2011; Sing et al. 2011; Knutson et al. 2012; Narita et al. 2013; Nascimbeni et al. 2015; Zellem et al. 2015). This approach is limited in two respects: (1) rotational variability monitoring traces only the nonaxisymmetric component of the stellar heterogeneity (Jackson \& Jeffries 2012), i.e., any persistent, underlying level of heterogeneity will not be detectable with variability monitoring; and (2) the source of the variability is commonly assumed to be a single giant spot, the size of which scales linearly with the variability amplitude, an assumption that provides only a lower limit on the extent of active regions.

Zellem et al. (2017) presented a novel method to remove relative changes in the stellar contribution to individual transits utilizing the out-of-transit data flanking each transit observation. The strength of this approach lies in that it does not require additional measurements to provide a relative correction for differences in spot and faculae covering fractions between observations. However, as with other variability-based techniques, this approach cannot correct for any persistent level 
of spots or faculae that may be present in all observations and can strongly alter transmission spectra (McCullough et al. 2014; Rackham et al. 2017).

Useful constraints on spot and faculae covering fractions are hindered by observational and theoretical limits on our knowledge of stellar photospheres. On the Sun, the disk passage of sunspots can produce relative declines in the solar total irradiance in the range of $\sim 0.1 \%-0.3 \%$ (e.g., Kopp et al. 2005). By contrast, field mid-to-late $M$ dwarfs $\left(M<0.35 M_{\odot}\right)$ with detectable rotation periods display rotational modulations with semi-amplitudes of $0.5 \%-1.0 \%$ (Newton et al. 2016), corresponding to peak-to-trough variability full amplitudes of $1 \%-2 \%$. Thus, variability amplitudes in $\mathbf{M}$ dwarfs are roughly an order of magnitude larger than those in the Sun.

Despite the clear importance of constraining spot and faculae covering fractions for exoplanet host stars, a systematic attempt to connect observed variabilities to covering fractions and thus stellar contamination signals is absent in the literature on transmission spectroscopy.

In this work, we employ a forward-modeling approach to explore the range of spot covering fractions consistent with observed photometric variabilities for field M-dwarf stars and their associated effects on visual and near-infrared $(0.3-5.5 \mu \mathrm{m})$ planetary transmission spectra. In Section 2 , we detail our model for placing constraints on spot and faculae covering fractions and their associated stellar contamination spectra. Section 3 provides the modeling results. We place our results in the context of observational attempts to constrain stellar heterogeneity and examine their impact on transmission spectra and density estimates of M-dwarf exoplanets in Section 4, including a focused discussion of the TRAPPIST1 system. Finally, we summarize our conclusions in Section 5.

\section{Methods}

\subsection{Synthetic Stellar Spectra}

We employed the PHOENIX (Husser et al. 2013) and DRIFT-PHOENIX (Witte et al. 2011) stellar spectral model grids to generate spectra for the immaculate photospheres, spots, and faculae of main-sequence $M$ dwarfs with spectral types from M0V to M9V. Both model grids are based on the stellar atmosphere code PHOENIX (Hauschildt \& Baron 1999), with the DRIFT-PHOENIX model grids including additional physics describing the formation and condensation of mineral dust clouds (Woitke \& Helling 2003, 2004; Helling \& Woitke 2006; Helling et al. 2008a, 2008b; Witte et al. 2009) that is applicable to late-M dwarfs and brown dwarfs. We considered models with solar metallicity $([\mathrm{Fe} / \mathrm{H}]=0.0)$ and no $\alpha$-element enrichment $([\alpha / \mathrm{Fe}]=0.0)$. We linearly interpolated between spectra in the grids to produce $0.3-5.5 \mu \mathrm{m}$ model spectra for the surface gravities and temperatures we required. The implicit assumption with this approach is that the emergent spectrum from distinct components of a stellar photosphere, such as the immaculate photosphere, spots, and faculae, can be approximated by models of disk-integrated stellar spectra of different temperatures. This approximation is commonly used in transit spectroscopy studies to constrain the contribution of unocculted photospheric heterogeneities to exoplanet transmission spectra (Pont et al. 2008, 2013; Sing et al. 2011, 2016; Huitson et al. 2013; Jordán et al. 2013; Fraine et al. 2014; Rackham et al. 2017). However, this simplification
Table 1

Adopted Stellar Parameters

\begin{tabular}{lcccc}
\hline \hline Sp. Type & $T_{\text {phot }}(\mathrm{K})$ & $T_{\text {spot }}(\mathrm{K})$ & $T_{\text {fac }}(\mathrm{K})$ & $\log g(\mathrm{cgs})$ \\
\hline M0V & 3800 & 3268 & 3900 & 4.7 \\
M1V & 3600 & 3096 & 3700 & 4.7 \\
M2V & 3400 & 2924 & 3500 & 4.8 \\
M3V & 3250 & 2795 & 3350 & 4.9 \\
M4V & 3100 & 2666 & 3200 & 5.3 \\
M5V & 2800 & 2408 & 2900 & 5.4 \\
M6V & 2600 & 2236 & 2700 & 5.6 \\
M7V & 2500 & 2150 & 2600 & 5.6 \\
M8V & 2400 & 2064 & 2500 & 5.7 \\
M9V & 2300 & 1978 & 2400 & 5.6 \\
\hline
\end{tabular}

Note. The photosphere temperature $T_{\text {phot }}$, spot temperature $T_{\text {spot, facula }}$ temperature $T_{\text {fac }}$, and surface gravity $\log g$ we adopt for each M-dwarf spectral type are listed.

neglects the dependence of the spectra of photospheric heterogeneities on magnetic field strength and limb distance, both of which modulate the emergent spectra of magnetic surface features (Norris et al. 2017). Nonetheless, we adopt the simplifying assumption of parameterizing component spectra by temperature and note that future efforts may benefit from the increased realism of 3D magnetohydrodynamics models.

Table 1 lists our adopted stellar parameters. For each spectral type, we calculated the surface gravity $g$ from the stellar masses and radii summarized by Kaltenegger \& Traub (2009) and adopted the stellar effective temperature from that same work as the photosphere temperature $T_{\text {phot }}$. Following Afram \& Berdyugina (2015), we adopted the relation $T_{\text {spot }}=0.86 \times$ $T_{\text {phot }}$, in which $T_{\text {spot }}$ is the spot temperature. We adopted the scaling relation $T_{\text {fac }}=T_{\text {phot }}+100 \mathrm{~K}$ (Gondoin 2008) for the facula temperature. Although there are uncertainties in the scaling relations of starspots and faculae, we do not expect our general results to be sensitive to the adopted relations. The temperature ranges of the spectral grids allowed us to simulate photosphere, spot, and facula spectra for spectral types M0VM5V with the PHOENIX model grid and M5V-M9V with the DRIFT-PHOENIX model grid.

\subsection{Spot Covering Fraction and Variability Amplitude Relation}

We explored the range of spot covering fractions consistent with an observed 1\% I-band variability full amplitude for each spectral type. We modeled the stellar photosphere using a rectangular grid with a resolution of $180 \times 360$ pixels. We initialized the model with an immaculate photosphere, setting the value of each resolution element to the flux of the photosphere spectrum integrated over the Bessel I-band response. $^{6}$ Likewise, when adding spots or faculae to the model, we utilized the integrated $I$-band fluxes of their respective spectra.

We considered four cases of stellar heterogeneities by varying two parameters: spot size and the presence or absence of faculae. In terms of spot size, we examined cases with smaller and larger spots, which we deem the "solar-like spots" and "giant spots" cases. In the solar-like spots case, each spot had a radius of $R_{\text {spot }}=2^{\circ}$, covering $400 \mathrm{ppm}$ of the projected

\footnotetext{
6 http://www.aip.de/en/research/facilities/stella/instruments/data/ johnson-ubvri-filter-curves
} 

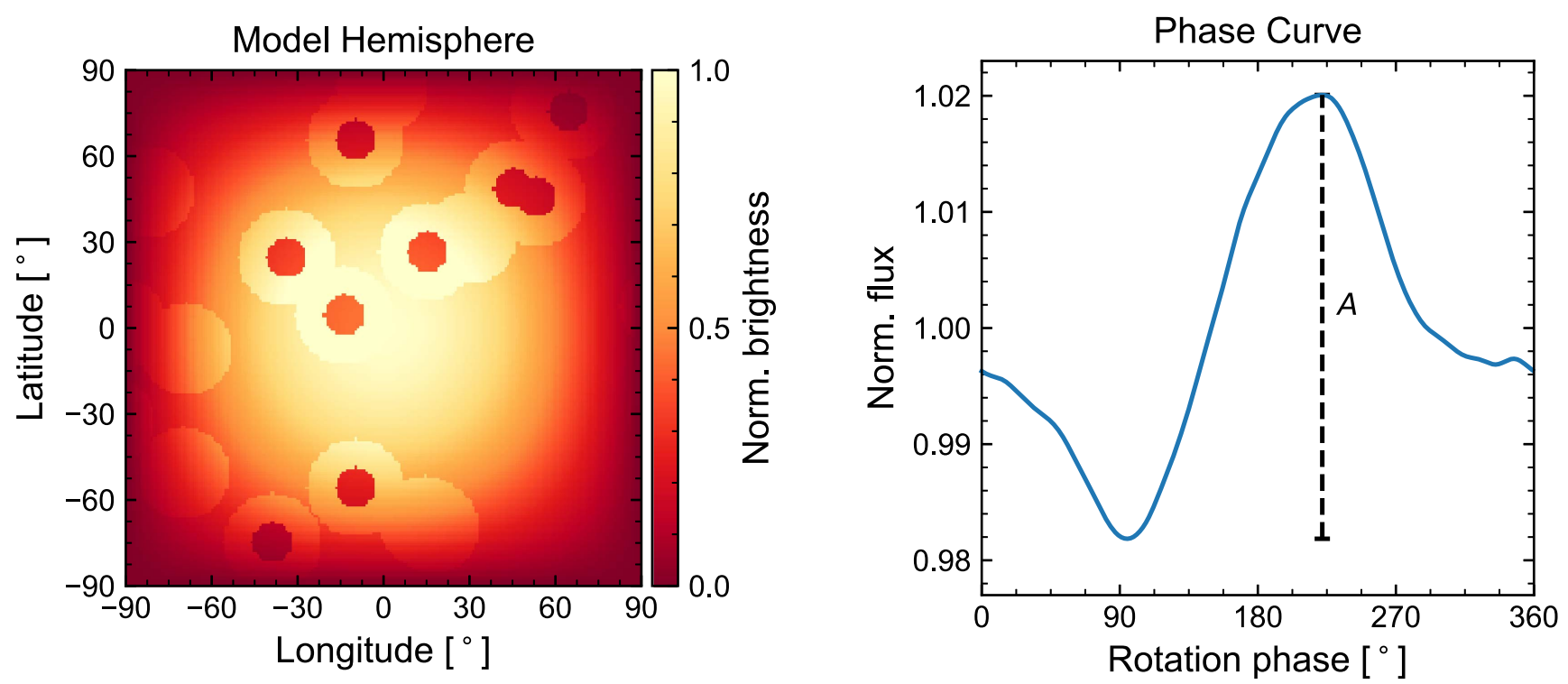

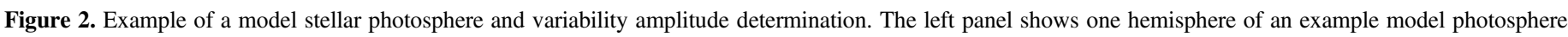



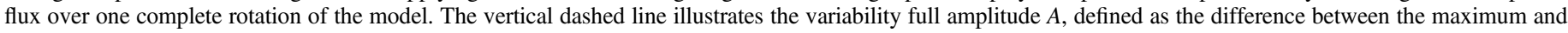
minimum normalized flux, which is $\sim 4 \%$ in this case.

hemisphere and representing a large spot group on the Sun (Mandal et al. 2017). In the giant spots case, each spot had a radius of $R_{\text {spot }}=7^{\circ}$, covering $5000 \mathrm{ppm}$ of the projected hemisphere and corresponding roughly to the largest spots detectable on active $\mathrm{M}$ dwarfs through molecular spectropolarimetry (Berdyugina 2011). For cases with faculae, we included faculae at a facula-to-spot area ratio of 10:1, following observations of the active Sun (Shapiro et al. 2014). Thus, the stellar heterogeneity cases we considered were the following: solar-like spots, giant spots, solar-like spots with faculae, and giant spots with faculae.

For each spectral type and stellar heterogeneity case, we examined the dependence of the variability on the spot covering fraction through an iterative process. In each iteration, we added a spot to the model photosphere at a randomly selected set of coordinates, ${ }^{7}$ recorded the spot covering fraction, and generated a phase curve. In cases including faculae, we added half of the facular area at positions adjacent to the spot and half in a roughly circular area at another randomly selected set of coordinates. We allowed spots to overwrite faculae but not vice versa in successive iterations to ensure the spot covering fraction increased monotonically. We generated a phase curve by applying a double cosine weighting kernel to one hemisphere of the rectangular grid $(180 \times 180$ pixels), summing the flux, and repeating the process for all 360 $x$-coordinates ("latitudes") in the model (Figure 2). We recorded the variability full amplitude $A$ as the difference between the minimum and maximum normalized flux in the phase curve at each iteration. This approach assumes that the stellar rotation axis is aligned well with the plane of the sky. As the presence of transits ensures that the planetary orbital plane is nearly edge-on, and obliquities between the stellar rotation axis and planetary orbital plane are generally $\lesssim 20^{\circ}$ (Winn et al. 2017),

\footnotetext{
7 We assumed no latitudinal dependence for the spot distribution. This assumption is good for active M-dwarf stars (Barnes et al. 2001; Barnes \& Collier Cameron 2001) but may not hold for earlier spectral types (Morris et al. 2017). We will examine the additional complication of latitudinal dependence of photospheric features in a future paper.
}

this assumption is good for most transiting exoplanet systems. Following this procedure, we iteratively added spots to the immaculate photosphere until reaching $50 \%$ spot coverage.

We repeated this procedure 100 times for a given set of stellar parameters and heterogeneity case to examine the central tendency and dispersion in modeling results. In each trial, we recorded the minimum spot covering fraction that produced a variability full amplitude of $1 \%(A=0.01)$. Using the results of the 100 trials, we calculated the mean spot covering fraction $f_{\text {spot,mean }}$ corresponding to $A=0.01$ and its standard deviation. We defined the spot covering fractions $1 \sigma$ below and above the mean as $f_{\text {spot,min }}$ and $f_{\text {spot, max }}$, respectively.

As spots were allowed to overwrite faculae in our model but not vice versa, the facula-to-spot area ratio drifted from its original 10:1 value as spots were added to the model. Thus, a distribution of faculae covering fractions existed for each spot covering fraction of interest. Accordingly, to quantify the central tendency and dispersion in results for models including faculae, we calculated the mean and standard deviation of faculae covering fractions in the 100 trials for each spot covering fraction of interest. We defined $f_{\text {fac,mean }}$ as the mean faculae covering fraction corresponding to $f_{\text {spot,mean }}, f_{\text {fac, min }}$ as the mean faculae covering fraction corresponding to $f_{\text {spot,min }}$ minus one standard deviation of that distribution, and $f_{\text {fac, max }}$ as the mean faculae covering fraction corresponding to $f_{\text {spot,max }}$ plus one standard deviation of that distribution.

\subsection{Model for Stellar Contamination Spectra}

Using the spot and faculae covering fractions determined through our variability modeling, we modeled the effect of stellar heterogeneity on observations of visual and near-infrared $(0.3-5.5 \mu \mathrm{m})$ exoplanet transmission spectra. We utilized the composite photosphere and atmospheric transmission model described in Rackham et al. (2017) and the spectra described in Section 2.1. Recasting Equation (11) of Rackham et al. (2017) 
in terms of transit depths and simplifying terms, we find

$$
D_{\lambda, \mathrm{obs}}=\frac{D_{\lambda}}{1-f_{\text {het }}\left(1-\frac{F_{\lambda, \text { het }}}{F_{\lambda, \text { phot }}}\right)},
$$

in which $D_{\lambda, \text { obs }}$ is the observed transit depth, $D_{\lambda}$ is the nominal transit depth (i.e., the square of the true wavelength-dependent planet-to-star radius ratio), $F_{\lambda \text {,phot }}$ is the spectrum of the photosphere, $F_{\lambda \text {,het }}$ is the spectrum of a photospheric heterogeneity (i.e., spots or faculae), and $f_{\text {het }}$ is the fraction of the projected stellar disk covered by the heterogeneity (see also McCullough et al. 2014, Equation (1)). This formalism assumes that the transit chord can be described well by $F_{\lambda \text {,phot }}$, which is the case for transits of an immaculate photosphere. It also applies to transit observations in which the amplitude of the spot- or faculae-crossing event is larger than the observational uncertainty, thus enabling the parameters of the photospheric heterogeneity to be modeled (e.g., SanchisOjeda \& Winn 2011; Huitson et al. 2013; Pont et al. 2013; Tregloan-Reed et al. 2013; Scandariato et al. 2017) or the affected portion of the light curve to be removed from the analysis (e.g., Pont et al. 2008; Carter et al. 2011; Narita et al. 2013).

The denominator on the right side of Equation (1) represents the signal imprinted on the observed transit depth by the stellar heterogeneity. It is a multiplicative change to the transit depth independent of the exoplanet transmission spectrum. Therefore, by dividing Equation (1) by $D_{\lambda}$, we can define the term

$$
\epsilon_{\lambda, \text { het }}=\frac{1}{1-f_{\text {het }}\left(1-\frac{F_{\lambda, \text { het }}}{F_{\lambda, \text { hot }}}\right)},
$$

which we refer to hereafter as the contamination spectrum. Approaching the problem in this way allows contamination spectra to be calculated for different stellar parameters and applied to any exoplanetary transmission spectrum of interest.

Equation (2) holds when the stellar disk can be described by two spectral components (i.e., immaculate photosphere and spots). In the case where spots and faculae are present in the stellar disk, the expression becomes

$$
\epsilon_{\lambda, s+f}=\frac{1}{1-f_{\text {spot }}\left(1-\frac{F_{\lambda, \text { spot }}}{F_{\lambda, \text { phot }}}\right)-f_{\text {fac }}\left(1-\frac{F_{\lambda, \text { fac }}}{F_{\lambda, \text { phot }}}\right)},
$$

where $\epsilon_{\lambda, s+f}$ is the contamination spectrum produced by the combination of unocculted spots and facular regions and the subscripts "spot" and "fac" refer to spots and faculae, respectively.

\section{Results}

\subsection{Spot Covering Fraction and Variability Amplitude Relation}

We find that, for all M-dwarf spectral types, an observed variability full amplitude corresponds to a typically wide range of spot covering fractions. Averaging over all spectral types, Table 2 provides a summary of the key results by heterogeneity case, including the spot and faculae covering fractions consistent with a $1 \%$ variability full amplitude, the average transit depth change over the full wavelength range studied, and the primary contributor (spots or faculae) to the stellar contamination spectrum.

For a given variability, we find that the spot covering fraction depends strongly on the spot size. Figure 3 illustrates the origin of this dependence using two examples of model photospheres with $f_{\text {spot }}=3 \%$. For a given spot covering fraction, the number density of spots is lower in the giant spots case than in the solar-like spots case, leading to more concentrated surface heterogeneities and larger variability signals. A single spot, for example, will always lead to rotational variability, while the variability signals from multiple spots positioned around the photosphere can add destructively, leading to a lower observed variability full amplitude. The solar-like spots case demonstrates this effect. It includes the same spot covering fraction as the giant spots case but produces a markedly lower level of variability. While chromospheric diagnostics may be used to distinguish active and quiet stars, variability monitoring can only place a lower limit on the spot covering fraction. In effect, the rotational variability full amplitude reflects only the nonaxisymmetric component of the stellar heterogeneity. The axisymmetric component, which can be larger than the nonaxisymmetric one, does not contribute to the observed variability but will affect transmission spectra.

Figure 4 shows the results of our variability amplitude modeling efforts for the spots-only case. For each spectral type and spot size, the relationship between the spot covering fraction and observed $I$-band variability full amplitude differs notably from a linear relation. Giant spots lead to overall larger variability full amplitudes than solar-like spots do for the same spot covering fraction. With the exception of variability full amplitudes $\leqslant 1 \%$ caused by giant spots, the linear relation is a poor approximation of the actual variability relation and underestimates the spot covering fraction. Instead, the variability full amplitude grows asymptotically as a function of spot covering fraction.

The shaded regions in Figure 4 illustrate the dispersion in the model outcomes. In effect, a given observed variability full amplitude corresponds to a range of spot covering fractions, which widens further for larger variability full amplitudes. Additionally, the solar-like spots case allows for still wider ranges of spot covering fractions. Table 3 provides the values of $f_{\text {spot,min }}, f_{\text {spot,mean }}$, and $f_{\text {spot,max }}$ (see Section 2.2) resulting from the set of 100 variability models we conducted for each spectral type.

Following the apparent square root dependence of the variability full amplitude $A$ on the spot covering fraction, we fit via least squares a scaling relation of the form

$$
A=C \times f_{\text {spot }}^{0.5}
$$

to each set of models, in which $C$ is a scaling coefficient that depends on both the spot contrast and size. We find that the relationship is approximated well by Equation (4) for all spotsonly models. In Table 3 , we provide the fitted values of $C$ with an uncertainty determined by the $68 \%$ dispersion in model outcomes (i.e., the shaded regions in Figure 4).

Figure 5 shows the results of the variability modeling for the spots-and-faculae case. In contrast to the spots-only case, the addition of faculae leads to larger I-band variability full amplitudes and a plateau in the relation for small spot covering fractions. As a result, a larger range of $f_{\text {spot }}$ corresponds to $A=0.01$ than in the spots-only case. Variability full 
Table 2

Summary of Variability Modeling Results by Heterogeneity Case

\begin{tabular}{lcccc}
\hline \hline \multirow{2}{*}{ Model Parameter } & \multicolumn{3}{c}{ Heterogeneity Case } \\
\cline { 2 - 5 } & Giant Spots & Solar-like Spots & Giant Spots + Faculae & Solar-like Spots + Faculae \\
\hline Spot covering fraction, $f_{\text {spot }}(\%)$ & $0.9_{-0.4}^{+1.3}$ & $12_{-6}^{+23}$ & $0.5_{-0.1}^{+0.8}$ & $14_{-7}^{+16}$ \\
Faculae covering fraction, $f_{\text {fac }}(\%)$ & $\ldots$ & $\ldots$ & $4.6_{-1.4}^{+7.4}$ & $63_{-25}^{+5}$ \\
Average transit depth change, $\bar{\epsilon}(\%)$ & 0.4 & 5.0 & -0.4 & 5.8 \\
Primary contributor to contamination spectrum & Spots & Spots & Faculae & Spots \\
\hline
\end{tabular}

Note. Here we summarize key results from the four cases of stellar photosphere heterogeneities that we considered (see Section 2.2). For each heterogeneity case, we provide the mean covering fractions of spots (and faculae, if possible) consistent with a $1 \% I$-band rotational variability across all spectral types considered. The error bars on these values refer to the means of the $f_{\text {spot,min }}, f_{\text {spot,max }}, f_{\text {fac, min }}$, and $f_{\text {fac, max }}$ parameters defined in Section 2.2. Also included is the average transit depth change produced by all models across the $0.3-5.5 \mu \mathrm{m}$ wavelength range and a qualitative assessment of the photospheric heterogeneity that dominates the contamination spectrum.

amplitudes begin to grow asymptotically again for $f_{\text {spot }}>0.1$ because the photospheres are nearly fully covered with faculae (due to the 10:1 faculae-to-spot ratio), and additional faculae do not contribute to the photospheric heterogeneity. Table 4 provides the range of $f_{\text {spot }}$ and $f_{\text {fac }}$ in the spots-and-faculae case for each spectral type.

For both spots-only and spots-and-faculae models, the variability modeling results show that extrapolations assuming a linear relation between the observed variability and spot covering fraction tend to underestimate the true spot covering fraction. Additionally, there is not a one-to-one relation between the observed variability and the spot covering fraction; instead, each observed variability full amplitude corresponds to a range of covering fractions.

\subsection{Stellar Contamination Spectra}

Figure 6 shows the contamination spectra produced by the spots-only models for three representative spectral types. The shaded regions illustrate the range of contamination spectra possible due to the range of spot covering fractions allowed. Unocculted spots lead to an increase in transit depths across the full wavelength range studied, with the largest increases at the shortest wavelengths. Variations in transit depth due to differences in the molecular opacities between the photosphere and spots are also apparent. They are strongest for mid-M dwarfs and overlap with many regions of interest for exoplanetary transmission features. We find that while the spectral type determines the specific features present, the overall scale of the contamination spectrum is largely independent of spectral type.

Spot size, however, has a strong effect on the scale of the contamination spectra for a given covering fraction. Considering the mean spot covering fractions consistent with a $1 \%$ variability full amplitude, giant spots alter transit depths by an average of $0.4 \%$ across $0.3-5.5 \mu \mathrm{m}$ wavelengths for M-dwarf spectral types. Given the allowed range of spot covering fractions, however, the average change could be as low as $0.2 \%$ or as high as $0.9 \%$. Solar-like spots, by contrast, produce much larger changes to transit depths due to the larger spot covering fractions present in this case. In this case, the average change to transit depths at these wavelengths for the mean spot covering fraction is $5.0 \%$ and could be as low as $2.6 \%$ or as high as $16 \%$, considering the allowed range of spot covering fractions.

Figure 7 shows contamination spectra produced by the spotsand-faculae variability models. In the giant spots case, the contamination spectra for all M-dwarf spectral types are dominated in the $0.3-5.5 \mu \mathrm{m}$ wavelength range by the facular contribution, which reduces transit depths at these wavelengths. Comparing Tables 3 and 4 shows that spot filling factors in all giant spots cases are very low, typically $\lesssim 1 \%$, but they are on average $2.2 \times$ smaller in the giant spots-and-faculae case than in the giant spots-only case. Thus, a general finding is that the facular component can dominate the visual and near-infrared contamination spectra for low spot covering fractions. On average, in the giant spots-and-faculae case, transit depths are decreased by $0.4 \%$ across this wavelength range for M-dwarf spectral types. Given the range of allowed spot and faculae covering fractions in these models, this average decrease could be as low as $0.3 \%$ or as high as $0.9 \%$. However, the contamination spectra of all M-dwarf spectral types are more pronounced at shorter wavelengths, producing a mean decrease in transit depth of $2.4 \%$ for $0.3-1 \mu \mathrm{m}$ wavelengths. We note that this result is consistent with the visual transmission spectrum of GJ 1214b (Rackham et al. 2017), which orbits a mid-M-dwarf host star.

The case of solar-like spots and faculae presents a challenge to our original assumption of an immaculate transit chord. The facular coverages determined in these models are typically $>50 \%$ and range as high as $72 \%$. If these facular coverages were indeed present, faculae would represent the dominant component of the stellar disk and likely the transit chord as well, precluding attempts to mask faculae crossings in light curves. Therefore, we assume in this case that the the faculae covering fraction within the transit chord is roughly equivalent to that of the unocculted stellar disk, and we calculate the contamination spectra shown in Figure 7 without the contribution from a faculae heterogeneity. Nonetheless, the range of faculae covering fractions presented in Table 4, particularly for late-M dwarfs, shows that facular coverages are largely unconstrained, and thus a wide range of facular contributions to stellar contamination spectra are possible for the solar-like spots case.

Regardless of the precise contribution from faculae, the addition of faculae to the heterogeneity model for the solar-like spots case generally increases the spot covering fractions that are consistent with an I-band variability full amplitude of $1 \%$ (Table 4). On average, spot covering fractions in the solar-like spots-and-faculae case are $1.2 \times$ larger than those in the case of solar-like spots only, leading to an average increase in transit depth across M-dwarf spectral types of $5.8 \%$ in the $0.3-$ $5.5 \mu \mathrm{m}$ wavelength range. 


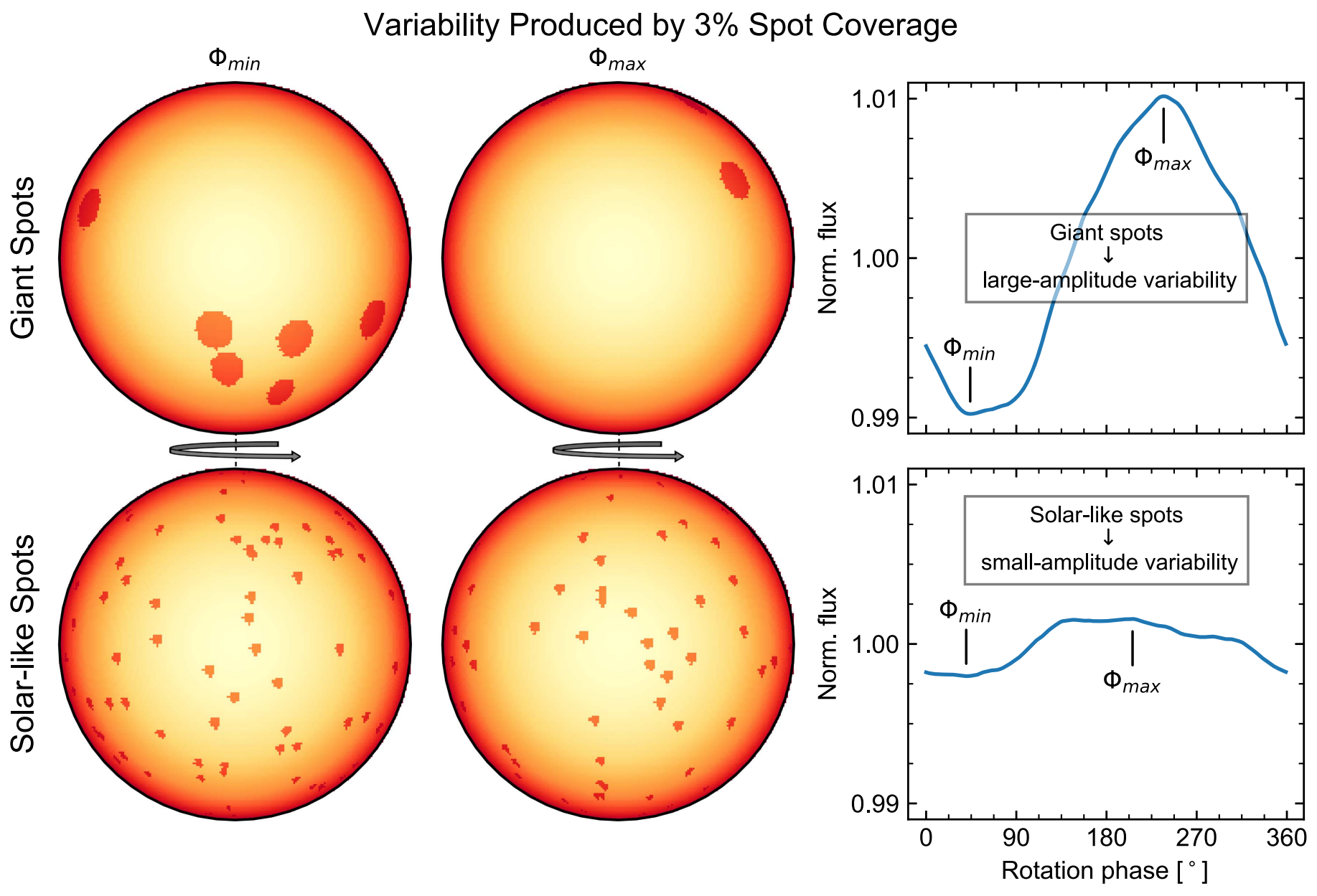

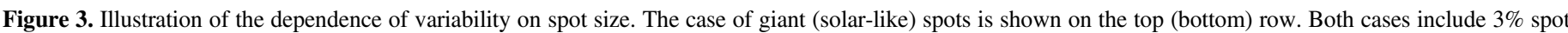

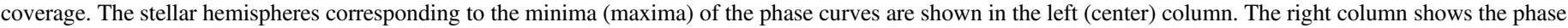
curves for the two cases, and the phases of the illustrated hemispheres are indicated.

\section{Discussion}

We find that, for a range of host star spectral types, heterogeneous stellar photospheres strongly alter transmission spectra. In the following sections, we compare the spot and faculae covering fractions determined by our modeling efforts with empirical results, discuss the effect of stellar contamination on derived planetary parameters, and examine the example of the TRAPPIST-1 system in detail.

\subsection{Comparison with Empirical Results}

Active-region properties such as fractional areal coverage are difficult to ascertain in spatially unresolved observations of stars. However, estimates can be made based on the unique properties of chromospheric line formation in the specific case of M-dwarf stars. In brief summary, the $\mathrm{H} \alpha$ line appears only weakly in absorption in the cool photospheres of $\mathrm{M}$ dwarfs. However, as demonstrated by Cram \& Mullan (1979), the onset of chromospheric heating first leads to an increase in absorption strength of the line. With further nonradiative heating, the line eventually attains a maximum in absorption equivalent width. Enhanced heating causes $\mathrm{H} \alpha$ to become collisionally controlled and driven into emission as the defining observational characteristic of a dMe star.

Based on this general behavior of $\mathrm{H} \alpha$ line formation in $\mathrm{M}$ dwarfs, Giampapa (1985) discussed how the observed equivalent width of $\mathrm{H} \alpha$ absorption yields estimates of the minimum area coverage of active regions. Since it is a chromospheric feature, its observed strength will be a function of its intrinsic absorption in plage and the total fractional area coverage of associated magnetically active facular regions. For specific M-dwarf (non-dMe) stars with measurements available at that time, ranging in spectral type from M1.5 to M5.5, he deduced model-independent filling factors of facular regions exceeding 10\%-26\%. A more stringent inference of minimum filling factor can be obtained from calculations of the maximum $\mathrm{H} \alpha$ absorption equivalent width attained in M-dwarf model chromospheres. Based on these computations (Cram \& Mullan 1979), Giampapa (1985) found a range in the minimum active-region filling factor of $31 \%-67 \%$ in the case of non-dMe stars characterized by $(R-I)_{K}=0.9$, corresponding to about $T_{\text {eff }}=3500 \mathrm{~K}$. Therefore, the fractional area coverage of faculae of even relatively quiescent $M$ dwarfs is widespread.

In the case of dMe stars, more intense chromospheric heating gives rise to $\mathrm{H} \alpha$ emission, which by itself does not lead to a direct estimate of facular area coverage. However, there is direct observational evidence for widespread, multi-kilogauss magnetic fields outside of spots in active $M$ dwarfs (i.e., dMe stars) at high filling factors in excess of $50 \%$ based on the analysis of magnetically sensitive photospheric features (Saar \& Linsky 1985; Reiners et al. 2009). Furthermore, the absence of any reported rotational modulation of $\mathrm{H} \alpha$ emission in $\mathrm{dMe}$ 


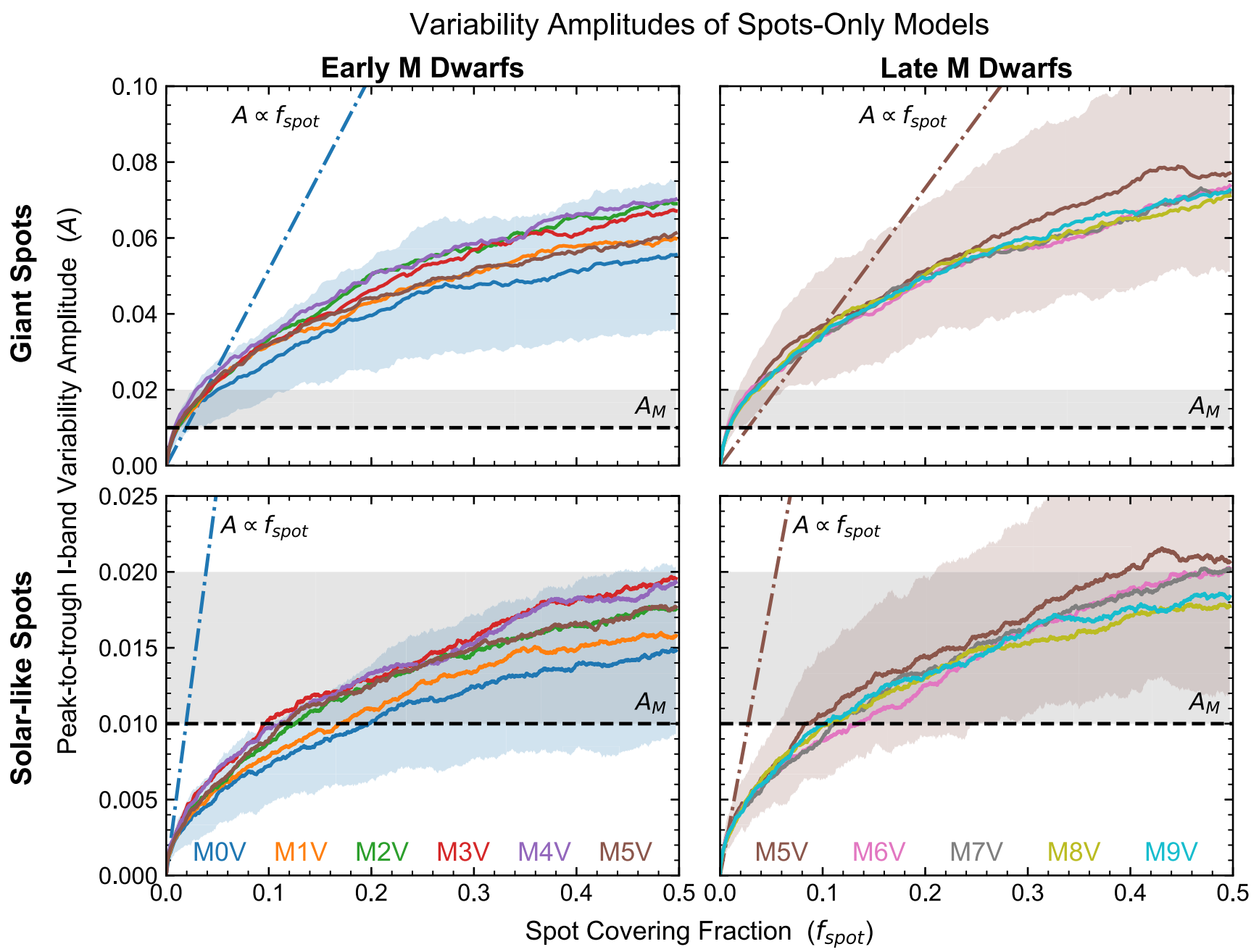

Figure 4. Variability full amplitudes as a function of spot covering fraction for the spots-only case. Results from the PHOENIX and DRIFT-PHOENIX models are shown in the left and right columns, respectively. Results using giant and solar-like spots are shown in the top and bottom rows, respectively. Note the difference in vertical scale in the upper and lower panels. Solid lines give the mean spot covering fraction for each spectral type, and shaded regions indicate the range encompassing $68 \%$ of the model outcomes for the earliest spectral type in each plot, which is comparable to the dispersion in model outcomes for all other spectral types. Additionally, the expected relation if variability were a linear function of spot covering fraction is shown by dash-dotted lines, given the $I$-band photosphere and spot fluxes of the earliest spectral type. The horizontal dashed lines show the TRAPPIST-1 variability full amplitude, and the gray shaded regions highlight the range of typical M-dwarf photometric modulations detected by Newton et al. (2016). For each case, the variability grows asymptotically as a function of $f_{\text {spot, }}$, and the linear relation generally underestimates $f_{\text {spot }}$. The dispersion in model outcomes demonstrates that a range of spot covering fractions corresponds to a given variability.

stars is consistent with the occurrence of an azimuthally symmetric, spatially widespread presence of facular regions on their surfaces.

In addition to active regions characterized by bright chromospheric emission or, in the case of quiescent $M$ dwarfs, chromospheric $\mathrm{H} \alpha$ absorption, the occurrence of periodic or quasi-periodic light-curve modulations in photometric bandpasses, indicative of the presence of cool spots, has an extensive record of observations. In a study of a compilation of extensive data sets, Newton et al. (2016, 2017) measured photometric semi-amplitudes in the range of roughly $0.5 \%-4 \%$ (Newton et al. 2017, Figure 9). We can therefore infer minimum starspot filling factors $f_{\text {spot }} \sim 1 \%-8 \%$ of the visible stellar disk in the case of completely black spots. Estimates of starspot temperatures provide a further refinement of the minimum spot filling factor. In an investigation of the magnetic properties of spots on the active, early-M dwarf AU Mic (dM1e), Berdyugina (2011) utilized polarimetric observations of temperature- and magnetically sensitive diatomic molecules to find spot temperatures $\sim 500-700 \mathrm{~K}$ cooler than the surrounding photosphere and with associated magnetic field strengths as high as $5.3 \mathrm{kG}$. With an effective temperature of $3775 \mathrm{~K}$ (Pagano et al. 2000), the ratio of spot-to-stellar effective temperature is in the range of $0.81-0.87$. In general, Afram \& Berdyugina (2015) found for $M$ dwarfs that spot models tend to have a spot-to-photosphere temperature ratio of 0.86 . Based on these measurements, for a semi-amplitude of $\sim 2 \%$ with a range of $T_{\text {spot }} / T_{\text {eff }}$ of $\sim 0.8-0.9$, we estimate spot filling factors in the range of $\sim 7 \%-12 \%$. Given that the photometric modulation depends on both contrast and departures from a longitudinally symmetric surface distribution of spots, this spot filling factor estimate should be regarded as a minimum value, since the observed light-curve modulation could arise from departures from axial symmetry in an otherwise widespread and uniform distribution of small spots. In fact, Jackson \& Jeffries (2013) demonstrated that smallamplitude light-curve modulation can arise from the combined effect of a random distribution of small spots characterized by a uniform size (i.e., scale length) and a plausible temperature contrast with the surrounding photosphere of 0.7. As an 
Table 3

Filling Factors and Scaling Coefficients Determined by Spots-only Models

\begin{tabular}{|c|c|c|c|c|c|}
\hline Sp. Type & Model Grid & $f_{\text {spot, } \min }$ & $f_{\text {spot,mean }}$ & $f_{\text {spot, } \max }$ & $C^{\mathrm{a}}$ \\
\hline \multicolumn{6}{|c|}{ Giant Spots } \\
\hline M0V & PHOENIX & 0.007 & 0.011 & 0.026 & $0.084 \pm 0.030$ \\
\hline M1V & PHOENIX & 0.006 & 0.011 & 0.029 & $0.092 \pm 0.036$ \\
\hline $\mathrm{M} 2 \mathrm{~V}$ & PHOENIX & 0.006 & 0.011 & 0.028 & $0.104 \pm 0.041$ \\
\hline M3V & PHOENIX & 0.006 & 0.010 & 0.022 & $0.100 \pm 0.038$ \\
\hline M4V & PHOENIX & 0.005 & 0.008 & 0.019 & $0.105 \pm 0.043$ \\
\hline M5V & PHOENIX & 0.006 & 0.010 & 0.023 & $0.092 \pm 0.035$ \\
\hline M5V & DRIFT-PHOENIX & 0.005 & 0.008 & 0.019 & $0.116 \pm 0.041$ \\
\hline M6V & DRIFT-PHOENIX & 0.005 & 0.008 & 0.015 & $0.106 \pm 0.040$ \\
\hline M7V & DRIFT-PHOENIX & 0.006 & 0.008 & 0.023 & $0.106 \pm 0.040$ \\
\hline M8V & DRIFT-PHOENIX & 0.006 & 0.009 & 0.023 & $0.106 \pm 0.041$ \\
\hline M9V & DRIFT-PHOENIX & 0.005 & 0.009 & 0.021 & $0.107 \pm 0.042$ \\
\hline \multicolumn{6}{|c|}{ Solar-like Spots } \\
\hline M0V & PHOENIX & 0.10 & 0.20 & 0.50 & $0.022 \pm 0.009$ \\
\hline M1V & PHOENIX & 0.08 & 0.17 & 0.46 & $0.024 \pm 0.009$ \\
\hline $\mathrm{M} 2 \mathrm{~V}$ & PHOENIX & 0.07 & 0.12 & 0.42 & $0.027 \pm 0.011$ \\
\hline M3V & PHOENIX & 0.06 & 0.10 & 0.30 & $0.029 \pm 0.010$ \\
\hline M4V & PHOENIX & 0.05 & 0.11 & 0.30 & $0.028 \pm 0.010$ \\
\hline M5V & PHOENIX & 0.07 & 0.11 & 0.43 & $0.027 \pm 0.010$ \\
\hline M5V & DRIFT-PHOENIX & 0.05 & 0.09 & 0.25 & $0.032 \pm 0.012$ \\
\hline M6V & DRIFT-PHOENIX & 0.06 & 0.13 & 0.28 & $0.029 \pm 0.010$ \\
\hline M7V & DRIFT-PHOENIX & 0.06 & 0.11 & 0.30 & $0.030 \pm 0.011$ \\
\hline M8V & DRIFT-PHOENIX & 0.05 & 0.11 & 0.30 & $0.028 \pm 0.011$ \\
\hline M9V & DRIFT-PHOENIX & 0.06 & 0.10 & 0.28 & $0.028 \pm 0.010$ \\
\hline
\end{tabular}

Note. Section 2.2 provides the definitions of the spot covering fractions $f_{\text {spot,min }}, f_{\text {spot,mean }}$, and $f_{\text {spot,max }}$.

${ }^{a}$ Scaling coefficient for square root scaling relation (Equation (4)).

illustration, their Monte Carlo simulations of light-curve amplitudes due to a large number of randomly distributed spots with, say, a characteristic length scale of 3.5 yield a mean amplitude of $1 \%$ with a filling factor of $\sim 30 \%$ (Jackson \& Jeffries 2013, Figure 4), in qualitative agreement with our variability modeling. Thus, in this context, we conclude that high filling factors of spots and faculae, such as those determined in this work, are a plausible interpretation of the observed light-curve modulations seen in $\mathrm{M}$ dwarfs.

\subsection{Stellar Contamination Mimicking and Masking Exoplanetary Features}

As shown in Figures 6 and 7, stellar heterogeneity can introduce significant spectral features in visual and nearinfrared transmission spectra. These deviations result from a difference in flux between the immaculate photosphere and heterogeneities such as spots and faculae (Equation (3)). Unocculted spots introduce positive features in transmission spectra that may be mistaken for evidence of absorption or scattering in the exoplanet atmosphere. By contrast, unocculted faculae introduce negative features, which can mask genuine spectral features originating in the exoplanet atmosphere.

Figure 8 illustrates the effect of stellar contamination at visual and near-infrared wavelengths of interest for molecular features in exoplanetary atmospheres. In general, stellar contamination increases transit depths and may mimic exoplanetary features, with the exception of the case of giant spots and faculae, in which the contribution from faculae dominates and may mask exoplanetary features. Transit depth changes are largest and therefore most problematic for (1) solar-like spots cases, (2) earlier spectral types, and (3) molecules with absorption bands at relatively short wavelengths, such as $\mathrm{O}_{2}$.

As a comparison for scale, the dashed horizontal line in Figure 8 indicates the $1.3 \%$ change in transit depth expected for a transiting Earth twin (atmospheric mean molecular weight $\mu=28.97 \mathrm{amu}$; equilibrium temperature $T_{\mathrm{eq}}=288 \mathrm{~K}$ ) due to an atmospheric feature covering five pressure scale heights. In the giant spots cases, stellar contamination can alter transit depths at overlapping wavelengths by a nonnegligible fraction of this amount, the effect of which is to boost the apparent size of features in the spots-only case and to weaken them in the giant spots-and-faculae case. Therefore, large unocculted spots can lead to a range of erroneous interpretations of transmission spectra: molecular abundances may appear enhanced or depleted, and the presence of a obscuring haze layer can be masked or mimicked.

By contrast, solar-like spots cases increase transit depths by much more than an expected exoplanetary feature. For early spectral types, the increase in transit depth can be more than 10 times that expected for planetary features. Such a strong feature could be easily identified as stellar in origin, though the scale of the feature, combined with the wide range of stellar contributions possible, would limit the accuracy of any determination of the underlying planetary feature. Later spectral types present a more pathological circumstance, as the magnitude of the transit depth change due to stellar contamination is comparable to that of a planetary atmospheric feature, allowing the signals to be easily mistaken. Therefore, constraining spot sizes and, by extension, spot covering fractions will be essential for investigations of atmospheric features in transmission spectra of low-mass exoplanets around 


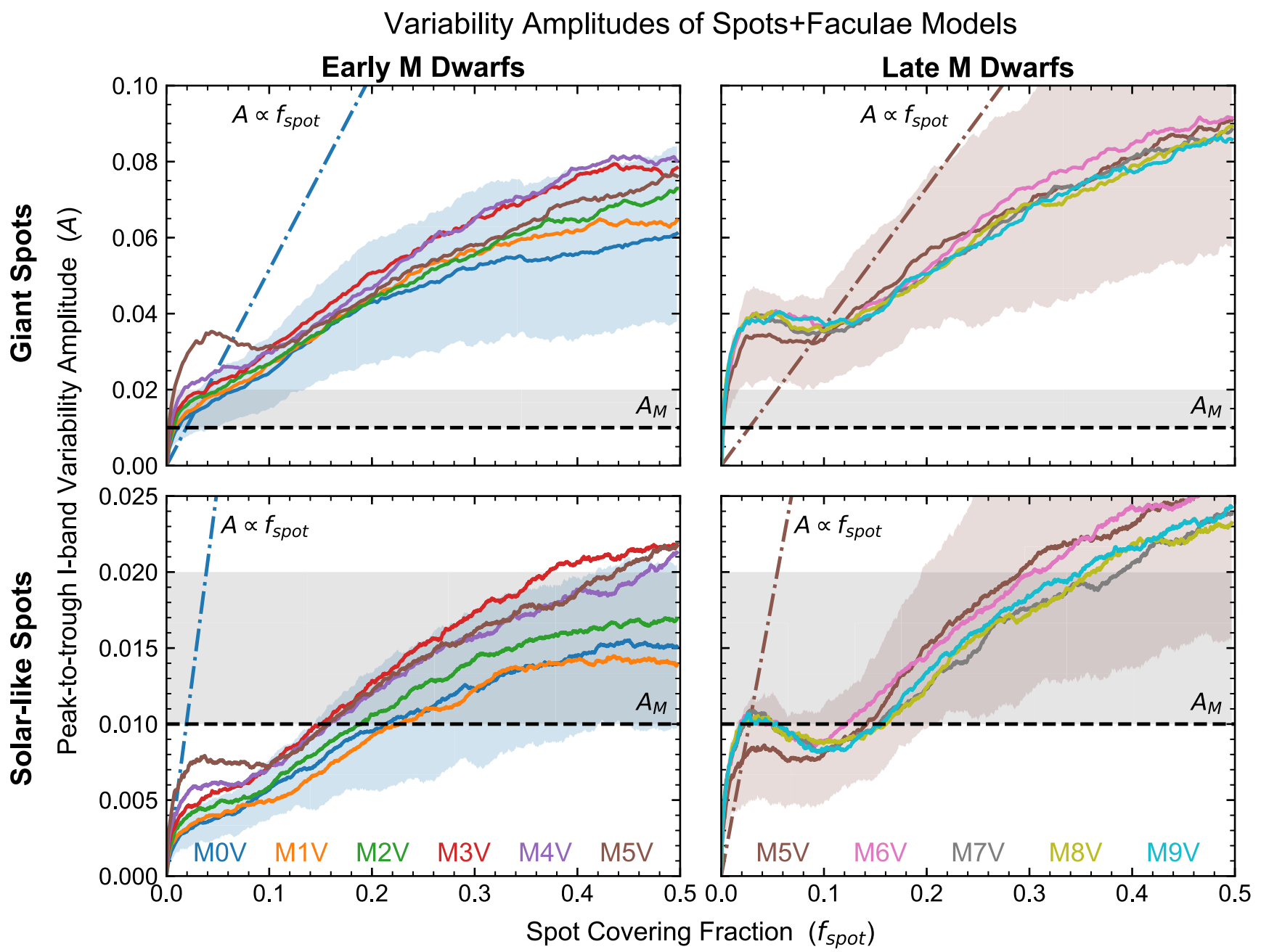

Figure 5. Variability full amplitudes as a function of spot covering fraction for the spots-and-faculae case. Note the difference in vertical scale in the upper and lower panels. Figure elements are the same as those in Figure 4. In contrast to the spots-only case, the addition of faculae in the variability modeling leads to larger variability amplitudes and a relative plateau in the relation for spot covering fractions $f_{\text {spot }}<0.1$.

late-M dwarfs, such as the TRAPPIST-1 system. We consider this topic in more detail in Section 4.5 below.

\subsection{Comparison to Observational Precisions}

Thus far we have discussed the effect of stellar heterogeneity on transmission spectra in terms of relative transit depth changes. This approach is useful because the multiplicative change in transit depth produced by unocculted spots and faculae can be applied to any planetary transmission spectrum. However, it is also informative to convert these relative transit depth changes to absolute ones that can be compared to observational precisions afforded by current and near-future facilities.

To do this, we must adopt parameters for the transiting exoplanet. At a minimum, these are the planetary radius and the expected scale of a planetary atmospheric feature. We consider two end-member cases for this comparison: a hot Neptune and an Earth twin. For the hot Neptune case, we adopt the parameters of GJ 436b (Butler et al. 2004; Gillon et al. 2007), which is one of the largest and hottest planets known to transit an $\mathrm{M}$ dwarf and represents a more readily observable exoplanet atmosphere. Its near-infrared transmission spectrum (Knutson et al. 2014) displays a weighted mean transit depth of $\bar{D}=0.70 \%$ and a $200 \mathrm{ppm}$ variation in transit depth (Knutson et al. 2014), which has been interpreted as an $\mathrm{H}_{2} \mathrm{O}$ absorption feature covering $0.46 \pm 0.25$ atmospheric scale heights (Crossfield \& Kreidberg 2017). This feature produces a relative transit depth change of

$$
\epsilon_{p}=\frac{\Delta D}{D}=\frac{2.0 \times 10^{-4}}{7.0 \times 10^{-3}}=2.9 \% .
$$

We adopt this observed relative transit depth change and the best-fit planetary radius of GJ 436b $\left(R_{p}=3.95 R_{\oplus}\right.$; Gillon et al. 2007) for the hot Neptune case. The other end-member case we consider is that of a transiting Earth twin $\left(R_{p}=1.0 R_{\oplus} ; \mu=28.97 \mathrm{amu} ; T_{\mathrm{eq}}=288 \mathrm{~K}\right)$, which produces a relative transit depth change of $\epsilon_{p}=1.3 \%$ for a five-scaleheight planetary feature and represents a larger observational challenge.

For each of these planetary cases, we calculate transit depths $D$ and absolute transit depth changes due to planetary atmospheric features $\Delta D_{p}$ as a function of M-dwarf spectral type using the stellar radii provided in Table 1 . We also calculate the mean absolute transit depth change due to stellar heterogeneity $\Delta D_{s}$ at wavelengths of interest for $\mathrm{H}_{2} \mathrm{O}$ absorption (as discussed in Section 4.2) for each of the four heterogeneity cases we consider. The results are summarized in 
Table 4

Filling Factors Determined by Spots-and-Faculae Models

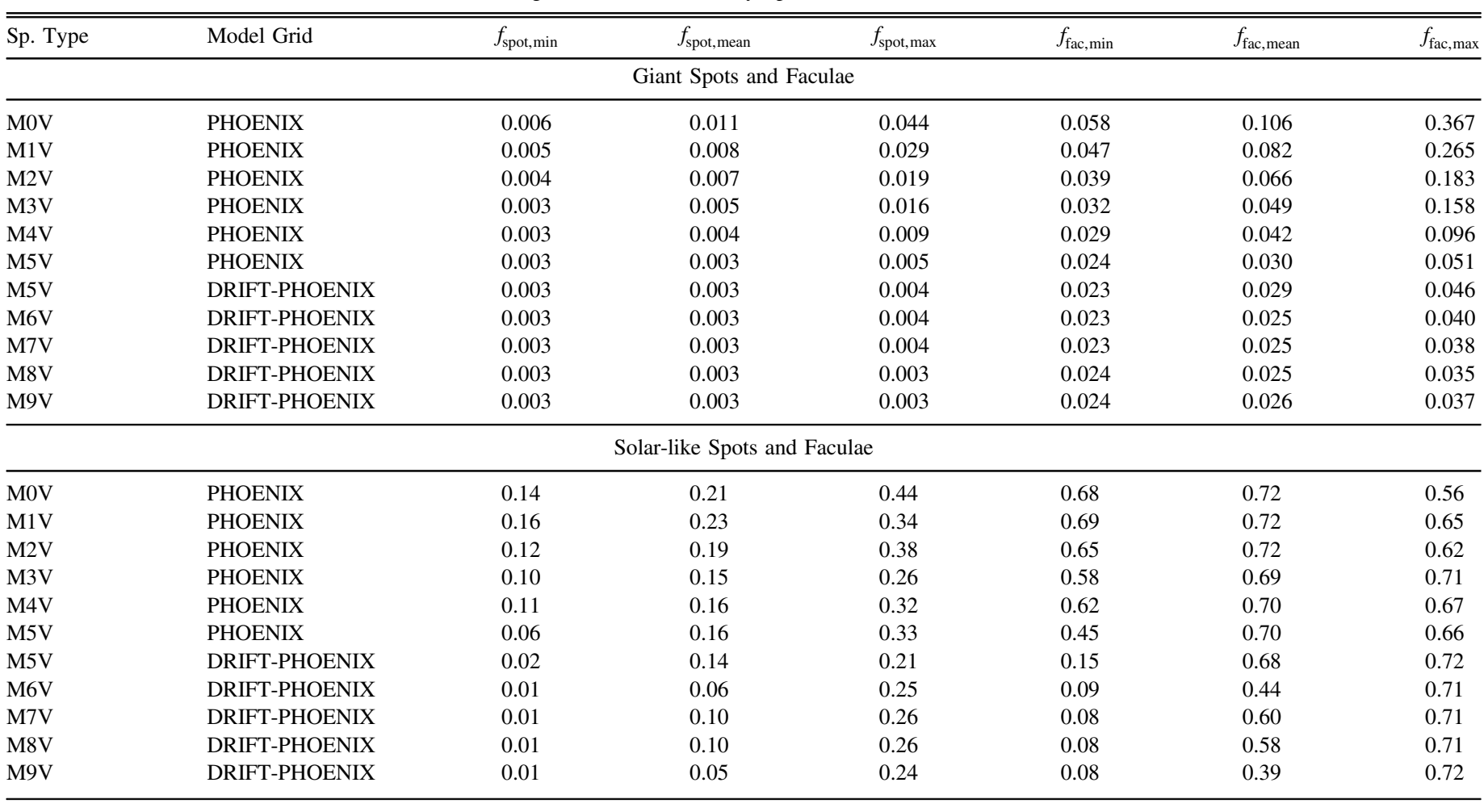

Note. Section 2.2 provides the definitions of the spot covering fractions $f_{\text {spot, min }}, f_{\text {spot,mean }}$, and $f_{\text {spot,max }}$ and the faculae covering fractions $f_{\text {fac, min }}, f_{\text {fac,mean }}$, and $f_{\text {fac,max }}$.

Tables 5 and 6 for the hot Neptune and Earth twin cases, respectively. Comparing these values of $\Delta D_{p}$ and $\Delta D_{s}$ suggests that for hot Neptunes, variations in transit depth due to the planetary atmosphere are roughly an order of magnitude larger than those due to stellar heterogeneity for giant spots cases. However, stellar signals are larger than planetary signals for all solar-like spots cases, with the largest difference between the signals for earlier spectral types. For Earth twins transiting $M$ dwarfs, in the case of giant spots, planetary signals are at least three times larger than stellar ones for all M-dwarf spectral types. However, as with the hot Neptune case, stellar signals due to solar-like spots are larger than planetary signals for all M-dwarf spectral types.

The question remains, however, of how these planetary and stellar signals compare to observational precisions. The most precise transmission spectrum obtained with HST/WFC3 to date contains a typical uncertainty of $30 \mathrm{ppm}$ on the transit depth in each wavelength channel (Kreidberg et al. 2014). This is similar to the noise floors for James Webb Space Telescope (JWST) instruments adopted by Greene et al. (2016) for wavelength ranges of interest in this study: $20 \mathrm{ppm}$ for NIRISS SOSS $(\lambda=1-2.5 \mu \mathrm{m})$ and $30 \mathrm{ppm}$ for NIRCam grism $(\lambda=2.5-5.0 \mu \mathrm{m})$. We adopt $30 \mathrm{ppm}$ as a fiducial noise floor for both HST and JWST observations. Considering this detection threshold, our results suggest that planetary atmospheric features are detectable for hot Neptunes orbiting $M$ dwarfs of all spectral types (Table 5). The effects of unocculted giant spots and facular regions are detectable for host stars with spectral types of roughly $\mathrm{M} 3 \mathrm{~V}$ and later, while in the more problematic case of solar-like spots, the effects of unocculted spots and faculae are detectable for all M-dwarf spectral types. In the case of a transiting Earth twin (Table 6), we estimate that planetary atmosphere features are detectable for spectral types M6V and later. The effects of unocculted giant spots and facular regions may alter the strength of these features by $\sim 20 \%$ but will only independently reach the detection threshold of $30 \mathrm{ppm}$ for M9V host stars. However, in the case of solar-like spots, we estimate that the effects of unocculted spots and faculae may be apparent in observations of spectral types as early as M3V or M4V.

In summary, we find that the most precise existing HST/ WFC3 G141 transmission spectra, as well as upcoming JWST transmission spectra of small planets around $\mathrm{M}$ dwarfs, can be significantly influenced by stellar contamination; any analysis of such spectra should consider the possible range of systematic contamination due to the transit light source effect.

\subsection{Systematic Errors in Density Measurements}

As the fundamental effect of starspots in this context is to influence the apparent radius of the planet relative to that of its star, we also explore here the impact of starspots on planet density measurements. Transiting planet surveys utilize visual and near-infrared bandpasses for discovery efforts (e.g., Nutzman \& Charbonneau 2008; Gillon et al. 2011; Jehin et al. 2011). With the exception of Kepler, which utilizes a broad, unfiltered visual bandpass, these surveys typically favor redder wavelengths such as the $I$ band in order to minimize the contribution of stellar variability to measurements. The bulk density of an exoplanet calculated from discovery transits can provide a clue as to their volatile content, making it one of the primary factors affecting whether a planet is selected for follow-up observations. Furthermore, the unfiltered Kepler photometry often remains the most precise transit depth measurement for most small planets, and therefore its accuracy 


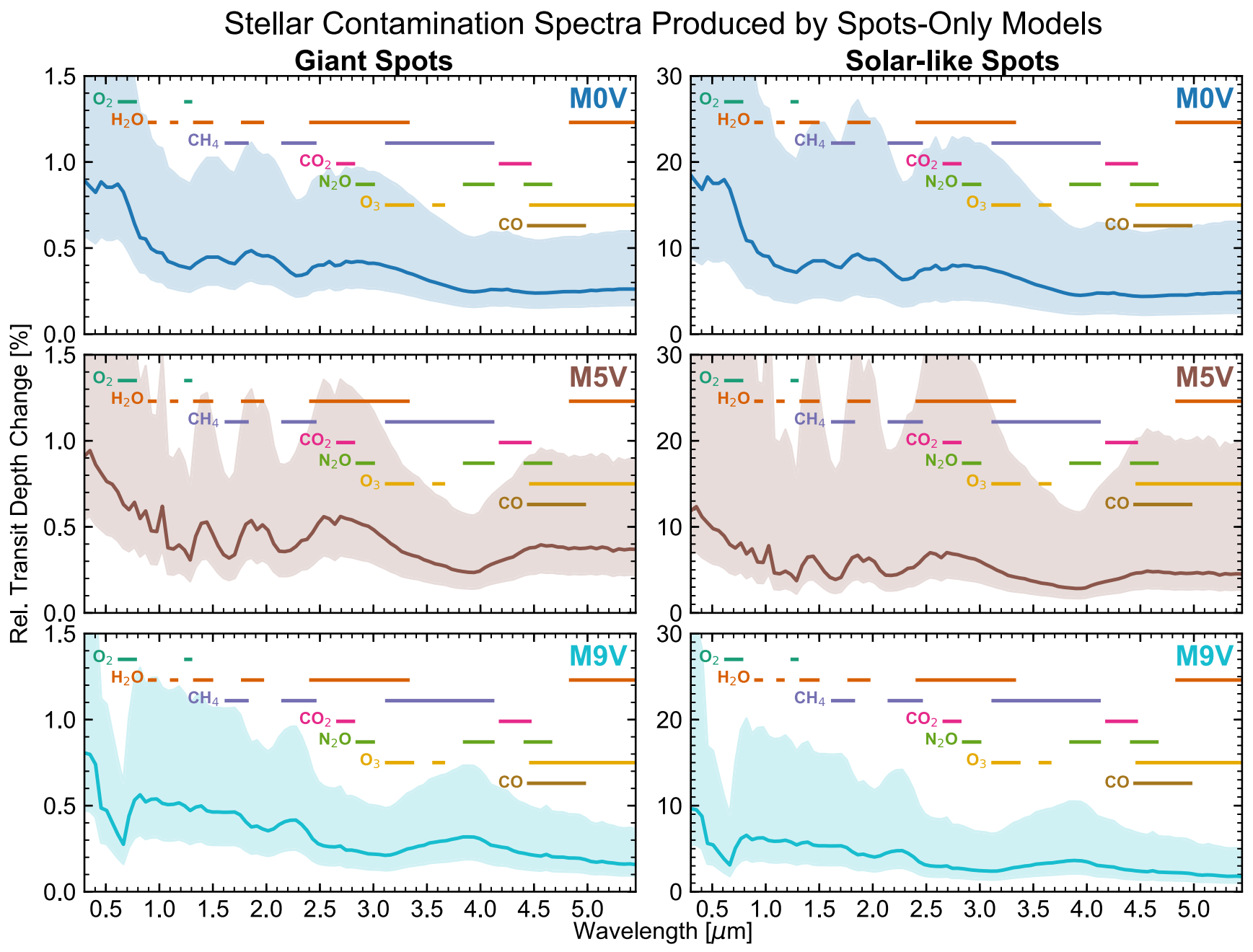

Figure 6. Stellar contamination spectra produced by spots-only models. Contamination spectra for early-, mid-, and late-M-dwarf spectral types are shown. Models for giant (solar-like) spots are shown in the left (right) column. Solid lines give the contamination spectrum for the mean spot covering fraction consistent with a $1 \%$ variability full amplitude. The shaded regions illustrate the range of contamination spectra produced by spot covering fractions consistent with that same variability (see Table 3). Overlapping wavelength bands for key exoplanetary atmospheric features are given.

affects inferences made about individual planets, as well as ensembles of planets.

Our work here, however, shows that the stellar contributions can be larger than previously suspected and may still contribute significantly even at red visual and near-infrared wavelengths, given the large range of stellar heterogeneity levels that may correspond to an observed rotational variability level. To determine the scale of the contribution, we calculated the range of radius and, by extension, density errors by integrating the contamination spectra presented in Section 3.2 over the Bessel I-band filter bandpass, which is similar to bandpasses used by ground-based transit surveys such as MEarth (Nutzman \& Charbonneau 2008) and TRAPPIST (Gillon et al. 2011; Jehin et al. 2011) and the Kepler ${ }^{8}$ and TESS (Ricker et al. 2015; Sullivan et al. 2015) spectral responses.

The resulting systematic radius and density errors are shown in Figure 9. Positive radius errors, which indicate that the apparent planetary radius is larger than the true planetary radius, correspond to negative density errors, which indicate that the apparent density is smaller than the true bulk density. We note that this systematic density error provides only a

\footnotetext{
https://archive.stsci.edu/kepler/fpc.html
}

minimum estimate of the total error in planetary bulk density, to which the observational uncertainty in radius and any error in mass estimate must be also be added.

As with the investigation into false planetary absorption features, we find the primary determinant of the systematic density error to be the spot size. Small spots cases are the most problematic, with an average density error of $13 \%$ across all spectral types and bandpasses that we considered and a maximum of $29 \%$ for M1 dwarfs in the Kepler bandpass. In the giant spots cases, by contrast, density errors are $1 \%$ on average, and the largest are 3\%. Aside from spot size, spectral type is the second-largest determinant of the systematic error. Earlier M-dwarf spectral types tend to produce density errors that are larger on average. Additionally, they demonstrate a larger dispersion in possible errors, as indicated by the error bars, given the wider range of spot and faculae covering fractions corresponding to an observed variability (see Tables 3 and 4). Finally, we find that the systematic errors are generally larger for bluer observational bandpasses, though the effect of the bandpass is smaller than that of either the spot size or host star spectral type.

To place the systematic radius errors in context, the difference in radius between an entirely rocky composition 


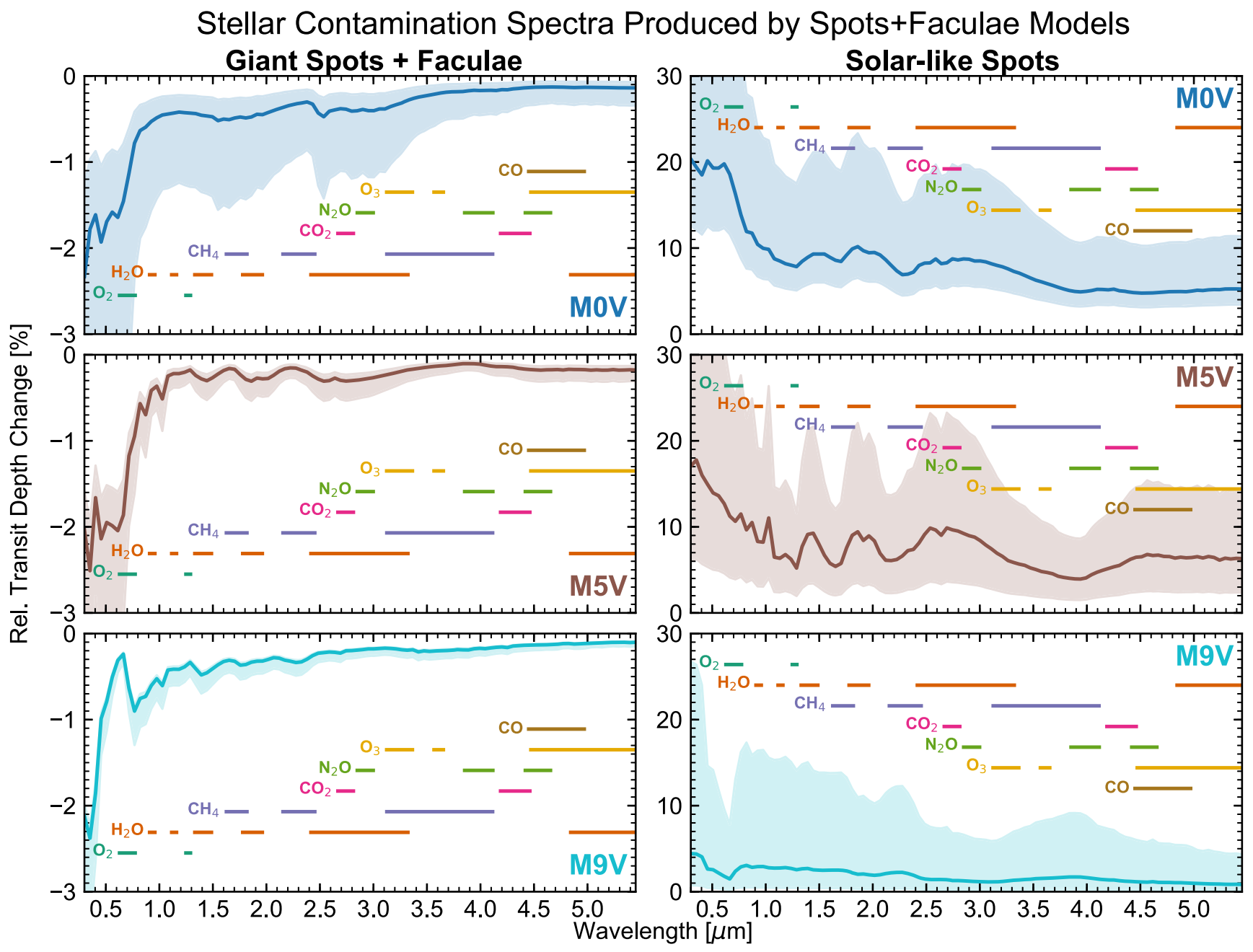

Figure 7. Stellar contamination spectra produced by spots-and-faculae models. The figure elements are the same as those in Figure 6 .

and a planet with $25 \% \mathrm{H}_{2} \mathrm{O}$ by mass for planets with masses between 0.125 and 32 Earth masses is 10\%-12\% (Zeng et al. 2016, Table 2), illustrated by the shaded regions in Figure 9. In terms of an observational comparison, we note that the $1 \sigma$ uncertainty on the radius of LHS $1140 \mathrm{~b}$ is $7.0 \%$ (Dittmann et al. 2017), giving it one of the best constrained densities of a sub-Neptune exoplanet to date. Therefore, while the effect of unocculted giant spots is easily hidden within observational uncertainties, unocculted solar-like spots can introduce a systematic error in radius that is comparable to the total error combined from the observational uncertainties in the radial velocity and transit photometry measurements and can significantly alter interpretations of the volatile content of an exoplanet. We note that this applies to M-dwarf planets that will be discovered by TESS, for which we predict sunspot-like spots will lead, on average, to systematic radii overestimates of $4.3 \%$ and density underestimates of $13 \%$.

\subsection{Application to TRAPPIST-1 System}

We utilized the approach detailed in Section 2 to place constraints on spot and faculae covering fractions for TRAPPIST-1 and their effects on observations of the TRAPPIST-1 planets. We opted to explore the case of the TRAPPIST-1 planets because they currently represent the best examples of habitable-zone, possibly rocky planets, and these planets are also likely to be primary targets for in-depth JWST transit spectroscopy.

\subsubsection{Spot and Faculae Covering Fractions}

We adopted the stellar parameters for TRAPPIST-1 from Gillon et al. (2017), including stellar mass, radius, effective temperature, and metallicity. With these parameters, we interpolated spectra covering $0.3-5.5 \mu \mathrm{m}$ from the DRIFTPHOENIX model grid. We set the photosphere temperature to the effective temperature and calculated the spot and faculae temperatures using the relations outlined in Section 2.1.

We integrated these spectra over the Bessel $I$-band response, similar to the $I+z$ bandpass utilized by the TRAPPIST survey (Gillon et al. 2016, 2017), to simulate the contributions of these spectral components to photometric observations. From visual inspection of Extended Data Figure 5 of Gillon et al. (2016), we estimate the variability full amplitude of TRAPPIST-1 to be $1 \%$.

Long-baseline monitoring of TRAPPIST-1 using the Spitzer Space Telescope, covering 35 transits, shows no definitive evidence of spot crossings (Gillon et al. 2017). Likewise, no spot crossings are apparent in existing HSTNWFC3 transit observations of TRAPPIST-1b and TRAPPIST-1c covering 1.1-1.7 $\mu \mathrm{m}$ with the G141 grism (de Wit et al. 2016), though the precision of G141 observations has allowed for the 


\section{Effect of Stellar Contamination on Planetary Absorption Features}

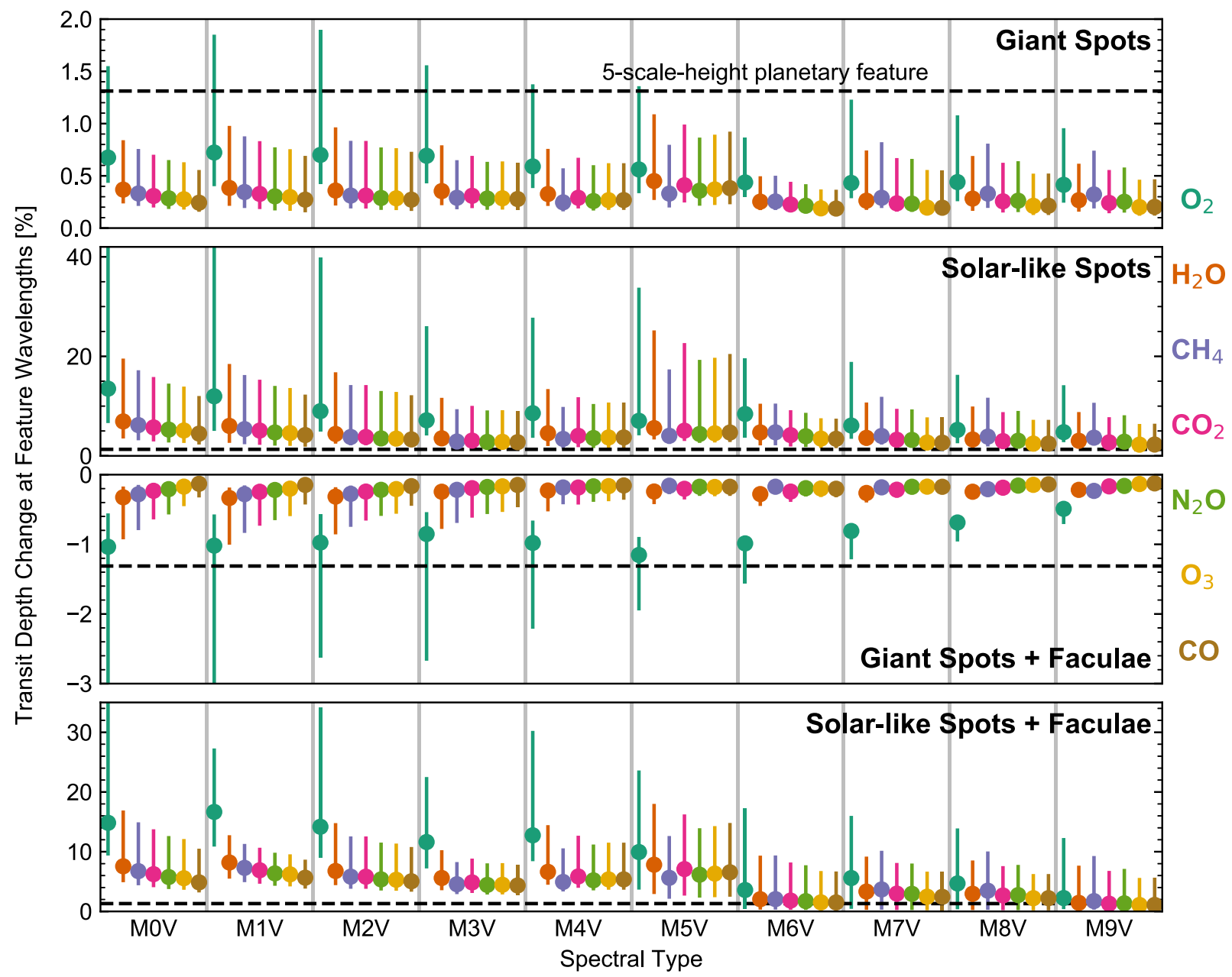

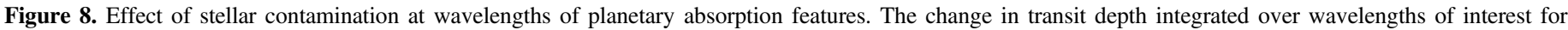

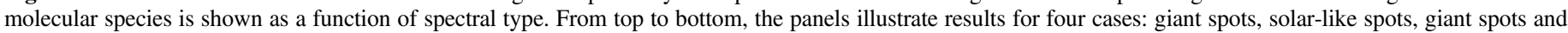

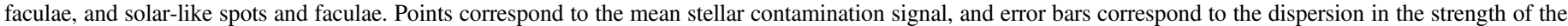

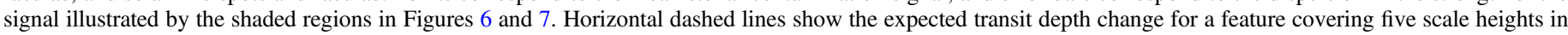
an Earth-like atmosphere, which is independent of stellar spectral type.

detection of large spot-crossing events in other M-dwarf transit observations (Kreidberg et al. 2014). Therefore, we assume that the presence of very large spots like those detected through molecular spectropolarimetry on active $\mathrm{M}$ dwarfs (Berdyugina 2011), each covering $0.5 \%$ of the projected stellar disk and comparable in size to the TRAPPIST-1 planets, is unlikely. Accordingly, we adopt a spot size of $2^{\circ}(400 \mathrm{ppm}$ or $0.04 \%$ of the projected stellar disk), typical of large spot groups on the Sun (Mandal et al. 2017). Given the radius of TRAPPIST-1 $(R=0.117 \pm 0.0036$; Gillon et al. 2017), this translates to spots with a radius of $R_{\text {spot }}=1.63 \pm 0.50 \times 10^{3} \mathrm{~km}$.

The left panel of Figure 10 displays the results of our variability modeling for both spots-only and spots-and-faculae cases. For both cases, we find that a large range of spot covering fractions is consistent with the observed variability full amplitude. We find $f_{\text {spot }}=11_{-6}^{+18} \%$ for the spots-only case, in which the quoted value is the mean spot covering fraction and the uncertainty refers to $68 \%$ dispersion in model results. For the spots-and-faculae case, we find $f_{\text {spot }}=8_{-7}^{+18} \%$ and $f_{\text {fac }}=54_{-46}^{+16} \%$. By contrast, under the assumption that the distribution of photospheric heterogeneities is fully asymmetric, in which case the variability amplitude would scale linearly with the spot covering fraction with a slope determined by the spot and photosphere I-band fluxes, one would infer a spot covering fraction of $1.0 \%$. We conclude that spot and faculae covering fractions for TRAPPIST-1 are largely unconstrained by the observed photometric variability amplitude, though the allowed range of fractions is typically much larger than one would infer by assuming a linear relation between spot covering fraction and variability amplitude.

\subsubsection{Stellar Contamination in Transmission Spectra}

The visual and near-infrared contamination spectra produced by the wide range of spots we infer for TRAPPIST-1 are shown in the center panel of Figure 10. The stellar contamination is a multiplicative effect and independent of the planetary transmission spectrum (Equation (2)). Thus, these relative transit depth changes are applicable to observations of each exoplanet in the TRAPPIST-1 system. As with the solar-like spot models in Figure 7, contributions from unocculted faculae are not included, given that faculae covering fractions in our models are typically $>50 \%$ and are therefore likely to be distributed homogeneously throughout the stellar disk. However, we calculate the contribution of unocculted spots using the spot 
Table 5

Transit Depths and Absolute Transit Depth Changes for a Representative Hot Neptune by Spectral Type

\begin{tabular}{lrrrrrr}
\hline \hline \multirow{2}{*}{ Sp. Type } & $D$ & \multirow{2}{*}{$\Delta D_{p}$} & \multicolumn{4}{c}{$\Delta D_{s}$ by Heterogeneity Case } \\
\cline { 4 - 7 } & & & & $\begin{array}{c}\text { Solar- } \\
\text { like }\end{array}$ & $\begin{array}{c}\text { Giant } \\
\text { Spots }+\end{array}$ & $\begin{array}{c}\text { Solar-like } \\
\text { Spots }+\end{array}$ \\
& & & $\begin{array}{c}\text { Giant } \\
\text { Spots }\end{array}$ & $\begin{array}{c}\text { Spots } \\
(\mathrm{ppm})\end{array}$ & $\begin{array}{c}\text { Faculae } \\
(\mathrm{ppm})\end{array}$ & $\begin{array}{c}\text { Faculae } \\
(\mathrm{ppm})\end{array}$ \\
\hline M0V & 3400 & 99 & 13 & $\mathbf{2 4 0}$ & -11 & $\mathbf{2 6 0}$ \\
M1V & 5400 & 160 & 21 & $\mathbf{3 3 0}$ & -18 & $\mathbf{4 5 0}$ \\
M2V & 6800 & 200 & 24 & $\mathbf{3 0 0}$ & -21 & $\mathbf{4 6 0}$ \\
M3V & 8600 & 250 & 30 & $\mathbf{3 0 0}$ & -21 & $\mathbf{4 8 0}$ \\
M4V & 19000 & 560 & 63 & $\mathbf{8 8 0}$ & -44 & $\mathbf{1 3 0 0}$ \\
M5V & 33000 & 950 & 100 & $\mathbf{1 1 0 0}$ & -85 & $\mathbf{1 9 0 0}$ \\
M6V & 58000 & 1700 & 150 & $\mathbf{2 7 0 0}$ & -160 & 1200 \\
M7V & 91000 & 2600 & 240 & $\mathbf{3 3 0 0}$ & -240 & $\mathbf{3 0 0 0}$ \\
M8V & 110000 & 3100 & 300 & $\mathbf{3 6 0 0}$ & -260 & $\mathbf{3 2 0 0}$ \\
M9V & 200000 & 5900 & 550 & $\mathbf{6 2 0 0}$ & -450 & 2900 \\
\hline
\end{tabular}

Note. Listed are the planetary transit depth $D$, transit depth change due to planetary atmospheric features $\Delta D_{p}$, and, for the four heterogeneity cases we consider, the transit depth change due to stellar heterogeneity $\Delta D_{s}$ (shown in bold for cases in which the stellar transit depth change is larger than that due to planetary atmospheric features). The scale of the stellar signal is smaller than that of a hot Neptune atmospheric feature for all giant spots cases, but it is generally larger than the planetary signal for cases with solar-like spots.

Table 6

Transit Depths and Absolute Transit Depth Changes for a Transiting Earth Twin by Spectral Type

\begin{tabular}{lcccccc}
\hline \hline \multirow{2}{*}{ Sp. Type } & \multirow{2}{*}{$D$} & $\Delta D_{p}$ & \multicolumn{4}{c}{$\Delta D_{s}$ by Heterogeneity Case } \\
\cline { 4 - 7 } & & & & $\begin{array}{c}\text { Solar- } \\
\text { like }\end{array}$ & $\begin{array}{c}\text { Giant } \\
\text { Spots }+\end{array}$ & $\begin{array}{c}\text { Solar-like } \\
\text { Spots }+\end{array}$ \\
& & & $\begin{array}{c}\text { Giant } \\
\text { Spots }\end{array}$ & $\begin{array}{c}\text { Spots } \\
(\mathrm{ppm})\end{array}$ & $\begin{array}{c}\text { Faculae } \\
(\mathrm{ppm})\end{array}$ & $\begin{array}{c}\text { Faculae } \\
(\mathrm{ppm})\end{array}$ \\
\hline M0V & 220 & 2.8 & 0.8 & $\mathbf{1 5}$ & -0.7 & $\mathbf{1 6}$ \\
M1V & 350 & 4.5 & 1.3 & $\mathbf{2 1}$ & -1.2 & $\mathbf{2 9}$ \\
M2V & 430 & 5.6 & 1.6 & $\mathbf{1 9}$ & -1.4 & $\mathbf{2 9}$ \\
M3V & 550 & 7.2 & 2.0 & $\mathbf{1 9}$ & -1.4 & $\mathbf{3 1}$ \\
M4V & 1200 & 16 & 4.0 & $\mathbf{5 6}$ & -2.8 & $\mathbf{8 2}$ \\
M5V & 2100 & 27 & 6.5 & $\mathbf{7 3}$ & -5.4 & $\mathbf{1 2 0}$ \\
M6V & 3700 & 48 & 9.4 & $\mathbf{1 8 0}$ & -10 & $\mathbf{7 6}$ \\
M7V & 5800 & 76 & 15 & $\mathbf{2 1 0}$ & -15 & $\mathbf{1 9 0}$ \\
M8V & 6900 & 90 & 20 & $\mathbf{2 3 0}$ & -17 & $\mathbf{2 1 0}$ \\
M9V & 13000 & 170 & 35 & $\mathbf{4 0 0}$ & -29 & $\mathbf{1 9 0}$ \\
\hline
\end{tabular}

Note. Listed are the planetary transit depth $D$, transit depth change due planetary atmospheric features $\Delta D_{p}$, and, for the four heterogeneity cases we consider, the transit depth change due to stellar heterogeneity $\Delta D_{s}$ (shown in bold for cases in which the stellar transit depth change is larger than that due to planetary atmospheric features). The scale of the stellar signal is smaller than that of an Earth twin atmospheric feature for all giant spots cases, but it is larger than the planetary signal for all cases with solar-like spots.

covering fractions determined by the spots-and-faculae models, which are generally smaller than those from the spots-only models, to exploit the increased realism of the spots-andfaculae models. The solid line shows the contamination spectrum corresponding to the mean spot covering fraction, and the shaded region indicates the range of spectra for the $68 \%$ confidence interval on $f_{\text {spot }}$. Compared to the contamination spectrum predicted by the fully asymmetric assumption, shown as a dash-dotted line, we find the level of stellar contamination to be roughly an order of magnitude larger.
Integrating over wavelengths of interest for key planetary molecular features, we illustrate the practical impact on observations in the right panel of Figure 10. For all molecules considered, we find the mean relative transit depth change to be comparable to or larger than that produced by an exoplanetary atmospheric feature. The shaded region in this panel illustrates the range of feature scales for the six innermost TRAPPIST-1 planets, calculated using the planetary parameters from Gillon et al. (2017) and assuming an Earth-like atmospheric mean molecular weight $(\mu=28.97 \mathrm{amu})$. They range from $0.8 \%$ for TRAPPIST-1g to $2.5 \%$ for TRAPPIST-1d. Given the transit depths of the TRAPPIST-1 planets, these correspond to absolute transit depth changes of $59 \mathrm{ppm}$ (TRAPPIST-1g) to $168 \mathrm{ppm}$ (TRAPPIST-1b). Assuming the same $30 \mathrm{ppm}$ detection threshold as in Section 4.3, atmospheric features with these scales are, in principle, detectable with both HST and JWST. In contrast to the planetary atmospheric signal, mean molecular feature errors produced by unocculted spots range from $2.1 \%$ for $\mathrm{CO}$ to $4.3 \%$ for $\mathrm{O}_{2}$. For the planet with the shallowest transit depth in this set, TRAPPIST-1d $(D=0.367 \% \pm$ $0.017 \%$; Gillon et al. 2017), a $2.1 \%$ relative change in transit depth corresponds to an absolute transit depth change of $77 \mathrm{ppm}$, which illustrates that the range of uncertainties we predict for all molecular features for the TRAPPIST-1 planets is above the fiducial $30 \mathrm{ppm}$ noise floor. In other words, we predict that stellar heterogeneity will significantly impact the interpretation of high-precision TRAPPIST-1 transmission spectra. Errors are most pronounced for molecules with absorption bands at shorter wavelengths. Large uncertainties exist for each molecular feature error due to the wide range of spot covering fractions and accompanying contamination spectra possible.

Finally, we calculate density errors for the TRAPPIST-1 planets due to stellar contamination. Integrating the contamination spectra presented in the center panel of Figure 10 over the $I$-band bandpass, we find an integrated systematic radius error of $\delta\left(R_{p}\right)=2.7_{-2.4}^{+6.8} \%$, in which the quoted value is the mean and the error refers to the $68 \%$ dispersion in modeling results. These values translate to density errors of $\Delta(\rho)=-8_{-20}^{+7} \%$; in other words, overestimating the planetary radius leads to an underestimate of the planetary density. Such a systematic error in density measurements could lead to overestimates of the volatile content of the TRAPPIST-1 planets. We provide updated densities for the six innermost TRAPPIST-1 planets in Table 7, adjusting the values reported by Gillon et al. (2017) for the density error due to stellar contamination. Our analysis suggests that stellar contamination may be partially responsible for the relatively low densities reported for planets in this system. More generally, this effect will be an important consideration when selecting targets for characterization follow-up from among a photometrically detected sample.

\section{Conclusions}

We have presented an examination of stellar contamination in visual and near-infrared $(0.3-5.5 \mu \mathrm{m})$ transmission spectra of M-dwarf exoplanets using model photospheres for M0-M9 dwarf stars with increasing levels of spots and faculae. Our key findings are the following:

1. For a given spot covering fraction, larger spots will produce a larger observed variability amplitude than that of smaller spots. Constraining the typical spot size for 


\section{Effect of Stellar Contamination on Planetary Density Calculation Kepler TESS I-band}

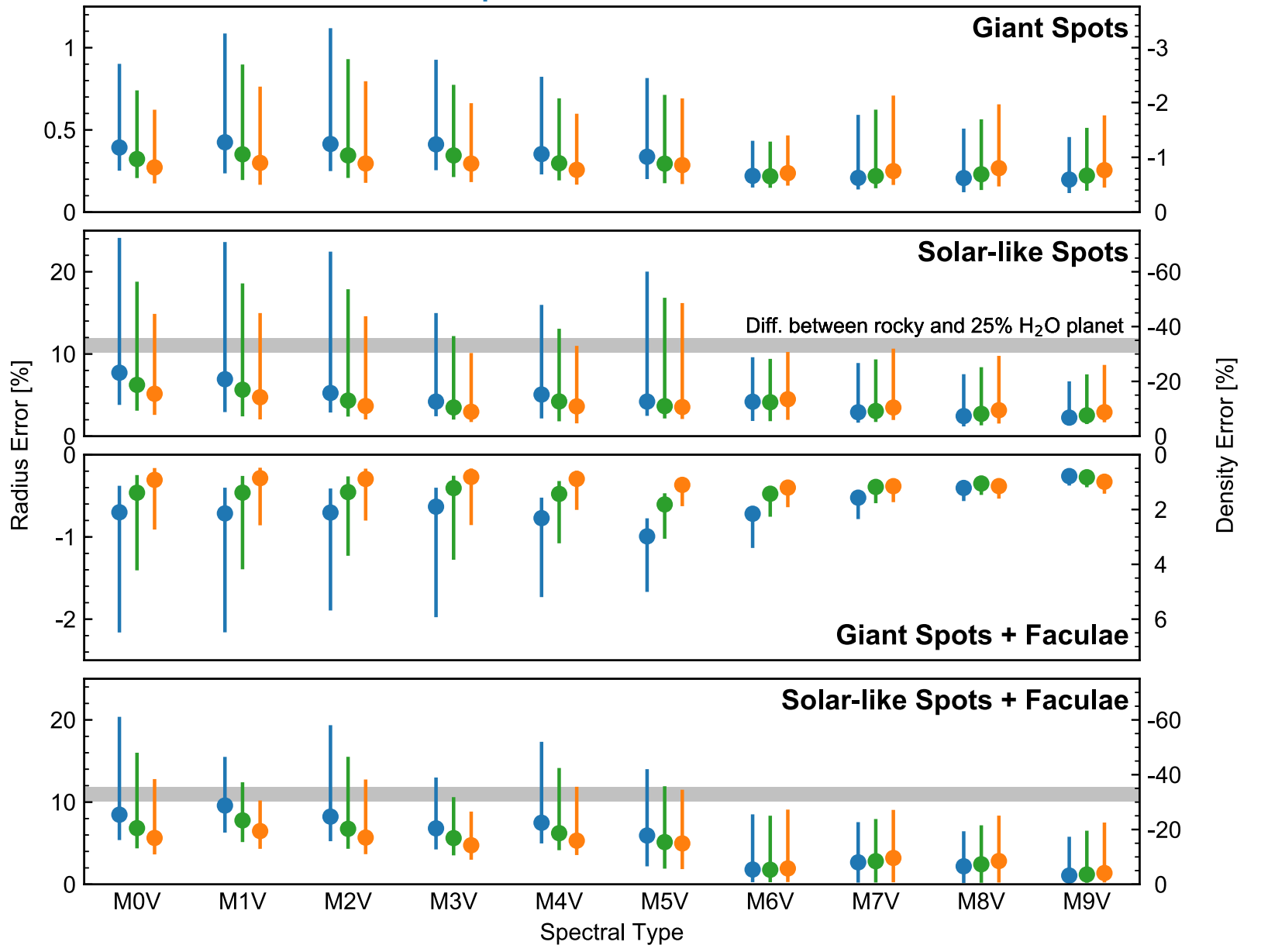

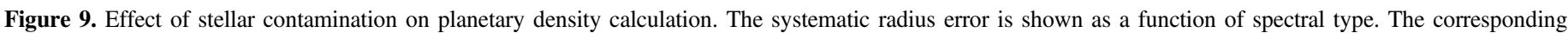

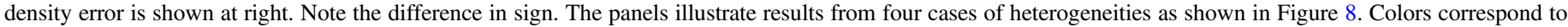

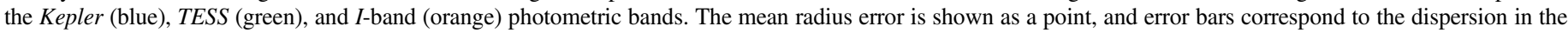

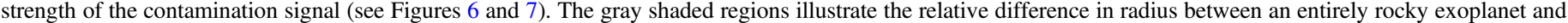
one with a significant volatile content for a range of masses between 0.125 and 32 Earth masses (see text).

exoplanet host stars is therefore crucial, since it will mediate the relationship between spot covering fraction and observed variability amplitude.

2. The relationship between spot covering fraction and observed variability amplitude is nonlinear, scaling generally like a square-root relation (Equation (4)) with a coefficient $0.02<C<0.11$ that depends on spot contrast and size. As such, previous corrections that have assumed a linear relationship between these variables likely underestimate the true spot covering fraction and, therefore, spectral contamination due to unocculted spots. We will explore this further in an upcoming paper.

3 . In contrast to the low levels of spot covering fractions $(\sim 1 \%)$ used in the literature, we show that a given variability amplitude corresponds to a wide range of spot and faculae covering fractions. For example, assuming spot sizes similar to those of sunspots, we find that a typical variability amplitude may correspond to spot covering fractions $0.14>f_{\text {spot }}>0.44$ and faculae covering fractions $0.56>f_{\text {fac }}>0.72$ for M0 dwarfs and $0.01>f_{\text {spot }}>0.24$ and $0.08>f_{\text {fac }}>0.72$ for M9 dwarfs. These wide ranges correspond to a similarly large uncertainty in the level of stellar contamination present in transmission spectra.

4. In stars with very large spots, the stellar contamination signal in the $0.3-5.5 \mu \mathrm{m}$ wavelength range can be dominated by the contribution from unocculted faculae, thereby decreasing observed transit depths. However, for stars with solar-like spots and the same observed variability, we find facular coverage to be so widespread that faculae represent the dominant component of the photosphere and are not likely to contribute to a heterogeneity signal.

5. Depending on spot size, we find that the stellar contamination signal can be more than $10 \times$ larger than the transit depth changes expected for atmospheric features in rocky exoplanets. Stellar contamination is most problematic for (1) stars with solar-like spots, (2) early-M-dwarf spectral types, and (3) molecules such as $\mathrm{O}_{2}$ with absorption bands at relatively short wavelengths.

6. We show that the stellar contamination is not limited to visual wavelengths but can also be very significant in the near-infrared bands, likely affecting upcoming JWST spectroscopy of transiting exoplanets. In the case of a 


\section{Stellar Contamination in the TRAPPIST-1 System}
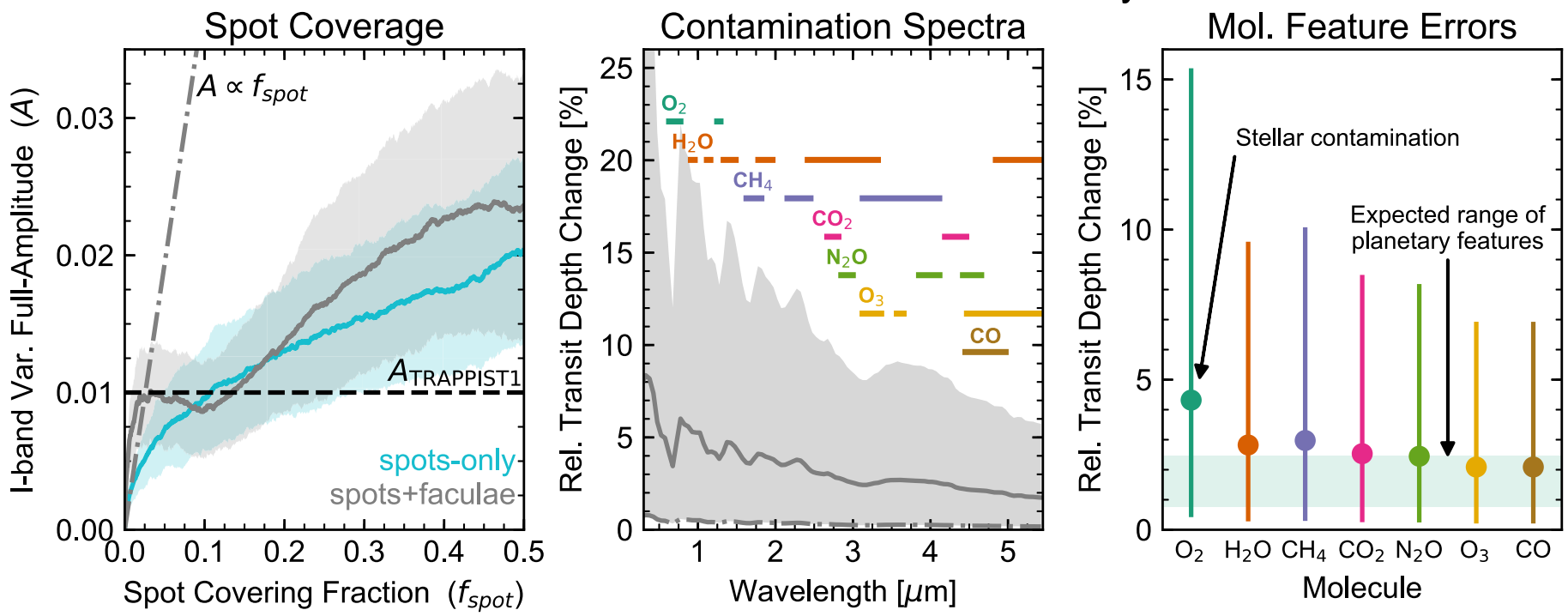

Figure 10. Summary of the effect of stellar heterogeneity in the TRAPPIST-1 system. The left panel shows I-band variability full amplitudes as a function of spot covering fraction for spots-only (teal) and spots-and-faculae (gray) cases. Results from solar-like spots models are shown. The plot elements are the same as those in Figure 4. The central panel shows the contamination spectra produced by spot covering fractions consistent with the observed variability of TRAPPIST-1. The plot elements are the same as those in Figure 6. The right panel shows the relative transit depth change at wavelengths of interest for key molecular features in exoplanet atmospheres. The shaded region illustrates the range of relative transit depth changes of interest for atmospheric features from the TRAPPIST-1 planets, assuming a five-scale-height feature in an Earth-like atmosphere.

Table 7

Revised Densities for the TRAPPIST-1 Planets

\begin{tabular}{lcc}
\hline \hline Planet & $\begin{array}{c}\text { Density from } \\
\text { Gillon et al. (2017) } \\
\left(\rho_{\oplus}{ }^{a}\right)\end{array}$ & $\begin{array}{c}\text { Revised Density } \\
\text { from This Work } \\
\left(\rho_{\oplus}\right)\end{array}$ \\
\hline $\mathrm{b}$ & $0.66 \pm 0.56$ & $0.68_{-0.58}^{+0.67}$ \\
$\mathrm{c}$ & $1.17 \pm 0.53$ & $1.21_{-0.57}^{+0.58}$ \\
$\mathrm{~d}$ & $0.89 \pm 0.60$ & $0.92_{-0.63}^{+0.73}$ \\
$\mathrm{e}$ & $0.80 \pm 0.76$ & $0.83_{-0.79}^{+0.90}$ \\
$\mathrm{f}$ & $0.60 \pm 0.17$ & $0.62_{-0.19}^{+0.23}$ \\
$\mathrm{~g}$ & $0.94 \pm 0.63$ & $0.97_{-0.66}^{+0.77}$ \\
\hline
\end{tabular}

Note. No mass and therefore density estimate for TRAPPIST-1h is available from Gillon et al. (2017).

${ }^{\mathrm{a}} \rho_{\oplus}=5.51 \mathrm{~g} \mathrm{~cm}^{-3}$.

transiting Earth twin, we find that unocculted giant spots can alter the strength of planetary absorption features in transmission spectra by $\sim 20 \%$ and unocculted spots and faculae can produce features greater than $30 \mathrm{ppm}$ for spectral types M3V and later.

7. We find that stellar spectral contamination across photometric bands due to unocculted starspots with sizes comparable to large sunspot groups can lead to significant errors in radius and therefore density, which may in turn lead to overestimates of planetary volatile content and introduce a stellar-type dependent bias in the density distribution of small planets. This result motivates a possible reassessment of mass-radius relationships derived from Kepler data, as well as calls for caution when interpreting upcoming broadband TESS photometrybased exoplanet density measurements.

8. In the case of the TRAPPIST-1 system, we find spot covering fractions $f_{\text {spot }}=8_{-7}^{+18} \%$ to be consistent with the variability reported by Gillon et al. (2016) when considering variability due to both spots and faculae. The associated stellar contamination signals in the optical and near-infrared alter transit depths at wavelengths of interest for planetary atmospheric species by roughly $1-15 \times$ the strength of the planetary feature, significantly complicating JWST follow-up observations of this system. Similarly, stellar contamination can cause the bulk densities of the TRAPPIST-1 planets to be underestimated by $8_{-7}^{+20} \%$, leading to overestimates of their volatile contents.

For TRAPPIST-1 and exciting M-dwarf exoplanet hosts in general, tighter constraints on spot and faculae covering fractions are crucial for correct interpretations of high-precision visual and near-infrared transmission spectra from low-mass exoplanets. Stellar contamination is likely to be a limiting factor for detecting biosignatures in transmission spectra of habitable-zone planets around $\mathrm{M}$ dwarfs. Conversely, exoplanet transit observations in multiple bands can be utilized as a spatial probe to infer the properties of stellar surface heterogeneities. This is particularly critical to achieving an accurate picture of the evolution of exoplanet system properties from young, active systems to those with older, more quiescent stellar hosts. In preparation for precise JWST observations probing for molecular features and potential biosignatures such as oxygen, water, and methane from small exoplanets orbiting M dwarfs, we encourage the community to work toward an equally precise understanding of the stellar photospheres providing the light source for transit observations.

B.R. acknowledges support from the National Science Foundation Graduate Research Fellowship Program under grant No. DGE-1143953. D.A. acknowledges support from the Max Planck Institute for Astronomy, Heidelberg, for a sabbatical visit. This research has made use of NASA's Astrophysics Data System and the Python modules SciPy, 
NumPy, and Matplotlib. The results reported herein benefited from collaborations and/or information exchange within NASA's Nexus for Exoplanet System Science (NExSS) research coordination network sponsored by NASA's Science Mission Directorate. The National Solar Observatory is operated by AURA under a cooperative agreement with the National Science Foundation.

\section{ORCID iDs}

Benjamin V. Rackham (1) https://orcid.org/0000-00023627-1676

Dániel Apai (1) https://orcid.org/0000-0003-3714-5855

Mark S. Giampapa (1) https://orcid.org/0000-0002-2132-5264

\section{References}

Afram, N., \& Berdyugina, S. V. 2015, A\&A, 576, A34

Andersen, J. M., \& Korhonen, H. 2015, MNRAS, 448, 3053

Babcock, H. W. 1961, ApJ, 133, 572

Barnes, J. R., \& Collier Cameron, A. 2001, MNRAS, 326, 950

Barnes, J. R., Collier Cameron, A., James, D. J., \& Donati, J.-F. 2001, MNRAS, 324, 231

Barstow, J. K., \& Irwin, P. G. J. 2016, MNRAS, 461, L92

Béky, B., Kipping, D. M., \& Holman, M. J. 2014, MNRAS, 442, 3686

Berdyugina, S. V. 2011, in ASP Conf. Ser. 437, Solar Polarization 6, ed. J. R. Kuhn et al. (San Francisco, CA: ASP), 219

Berta, Z. K., Charbonneau, D., Bean, J., et al. 2011, ApJ, 736, 12

Berta-Thompson, Z. K., Irwin, J., Charbonneau, D., et al. 2015, Natur, 527, 204

Bolmont, E., Selsis, F., Owen, J. E., et al. 2017, MNRAS, 464, 3728

Brown, T. M. 2001, ApJ, 553, 1006

Butler, R. P., Vogt, S. S., Marcy, G. W., et al. 2004, ApJ, 617, 580

Carter, J. A., Winn, J. N., Holman, M. J., et al. 2011, ApJ, 730, 82

Cauley, P. W., Redfield, S., \& Jensen, A. G. 2017, AJ, 153, 217

Cram, L. E., \& Mullan, D. J. 1979, ApJ, 234, 579

Crossfield, I. J. M., \& Kreidberg, L. 2017, AJ, 154, 261

Désert, J.-M., Sing, D., Vidal-Madjar, A., et al. 2011, A\&A, 526, A12

de Wit, J., Wakeford, H. R., Gillon, M., et al. 2016, Natur, 537, 69

Dittmann, J. A., Close, L. M., Green, E. M., \& Fenwick, M. 2009, ApJ, 701, 756

Dittmann, J. A., Irwin, J. M., Charbonneau, D., et al. 2017, Natur, 544, 333

Dravins, D., Ludwig, H.-G., Dahlén, E., \& Pazira, H. 2017a, A\&A, 605, A90

Dravins, D., Ludwig, H.-G., Dahlén, E., \& Pazira, H. 2017b, A\&A, 605, A91

Fraine, J., Deming, D., Benneke, B., et al. 2014, Natur, 513, 526

Giampapa, M. S. 1985, ApJ, 299, 781

Gillon, M., Jehin, E., Lederer, S. M., et al. 2016, Natur, 533, 221

Gillon, M., Jehin, E., Magain, P., et al. 2011, EPJWC, 11, 06002

Gillon, M., Pont, F., Demory, B.-O., et al. 2007, A\&A, 472, L13

Gillon, M., Triaud, A. H. M. J., Demory, B.-O., et al. 2017, Natur, 542, 456 Gondoin, P. 2008, A\&A, 478, 883

Goulding, N. T., Barnes, J. R., Pinfield, D. J., et al. 2012, MNRAS, 427, 3358

Greene, T. P., Line, M. R., Montero, C., et al. 2016, ApJ, 817, 17

Hauschildt, P. H., \& Baron, E. 1999, JCoAM, 109, 41

Helling, Ch., Dehn, M., Woitke, P., \& Hauschildt, P. H. 2008a, ApJ, 675, L105 Helling, Ch., \& Woitke, P. 2006, A\&A, 455, 325

Helling, Ch., Woitke, P., \& Thi, W.-F. 2008b, A\&A, 485, 547
Huitson, C. M., Sing, D. K., Pont, F., et al. 2013, MNRAS, 434, 3252

Husser, T.-O., Wende-von Berg, S., Dreizler, S., et al. 2013, A\&A, 553, A6

Jackson, R. J., \& Jeffries, R. D. 2012, MNRAS, 423, 2966

Jackson, R. J., \& Jeffries, R. D. 2013, MNRAS, 431, 1883

Jehin, E., Gillon, M., Queloz, D., et al. 2011, Msngr, 145, 2

Jordán, A., Espinoza, N., Rabus, M., et al. 2013, ApJ, 778, 184

Kaltenegger, L., \& Traub, W. A. 2009, ApJ, 698, 519

Knutson, H. A., Benneke, B., Deming, D., \& Homeier, D. 2014, Natur, 505, 66

Knutson, H. A., Lewis, N., Fortney, J. J., et al. 2012, ApJ, 754, 22

Kopp, G., Lawrence, G., \& Rottman, G. 2005, SoPh, 230, 129

Kreidberg, L., Bean, J. L., Désert, J.-M., et al. 2014, Natur, 505, 69

Llama, J., Jardine, M., Mackay, D. H., \& Fares, R. 2012, MNRAS, 422, L72

Llama, J., \& Shkolnik, E. L. 2015, ApJ, 802, 41

Llama, J., \& Shkolnik, E. L. 2016, ApJ, 817, 81

Luger, R., Sestovic, M., Kruse, E., et al. 2017, NatAs, 1, 0129

Mandal, S., Hegde, M., Samanta, T., et al. 2017, A\&A, 601, A106

Maunder, E. W. 1922, MNRAS, 82, 534

McCullough, P. R., Crouzet, N., Deming, D., \& Madhusudhan, N. 2014, ApJ, 791,55

Miller-Ricci, E., Seager, S., \& Sasselov, D. 2009, ApJ, 690, 1056

Morris, B. M., Hebb, L., Davenport, J. R. A., Rohn, G., \& Hawley, S. L. 2017, ApJ, 846, 99

Narita, N., Fukui, A., Ikoma, M., et al. 2013, ApJ, 773, 144

Nascimbeni, V., Mallonn, M., Scandariato, G., et al. 2015, A\&A, 579, A113

Newton, E. R., Irwin, J., Charbonneau, D., et al. 2017, ApJ, 834, 85

Newton, E. R., Irwin, J., Charbonneau, D., Berta-Thompson, Z. K., \& Dittmann, J. A. 2016, ApJL, 821, L19

Norris, C. M., Beeck, B., Unruh, Y. C., et al. 2017, A\&A, 605, A45

Nutzman, P., \& Charbonneau, D. 2008, PASP, 120, 317

Oshagh, M., Santos, N. C., Ehrenreich, D., et al. 2014, A\&A, 568, A99

Pagano, I., Linsky, J. L., Carkner, L., et al. 2000, ApJ, 532, 497

Pont, F., Knutson, H., Gilliland, R. L., Moutou, C., \& Charbonneau, D. 2008, MNRAS, 385, 109

Pont, F., Sing, D. K., Gibson, N. P., et al. 2013, MNRAS, 432, 2917

Rackham, B., Espinoza, N., Apai, D., et al. 2017, ApJ, 834, 151

Reiners, A., Basri, G., \& Browning, M. 2009, ApJ, 692, 538

Ricker, G. R., Winn, J. N., Vanderspek, R., et al. 2015, JATIS, 1, 014003

Saar, S. H., \& Linsky, J. L. 1985, ApJL, 299, L47

Sanchis-Ojeda, R., \& Winn, J. N. 2011, ApJ, 743, 61

Scandariato, G., Nascimbeni, V., Lanza, A. F., et al. 2017, A\&A, 606, A134

Seager, S., \& Sasselov, D. D. 2000, ApJ, 537, 916

Shapiro, A. I., Solanki, S. K., Krivova, N. A., et al. 2014, A\&A, 569, A38

Sing, D. K., Fortney, J. J., Nikolov, N., et al. 2016, Natur, 529, 59

Sing, D. K., Pont, F., Aigrain, S., et al. 2011, MNRAS, 416, 1443

Sullivan, P. W., Winn, J. N., Berta-Thompson, Z. K., et al. 2015, ApJ, 809, 77

Tregloan-Reed, J., Southworth, J., \& Tappert, C. 2013, MNRAS, 428, 3671

Vida, K., Kövári, Z., Pál, A., Oláh, K., \& Kriskovics, L. 2017, ApJ, 841, 124

Wheatley, P. J., Louden, T., Bourrier, V., Ehrenreich, D., \& Gillon, M. 2017, MNRAS, 465, L74

Winn, J. N., Petigura, E. A., Morton, T. D., et al. 2017, AJ, 154, 270

Witte, S., Helling, Ch., Barman, T., Heidrich, N., \& Hauschildt, P. 2011, A\&A, 529, A44

Witte, S., Helling, Ch., \& Hauschildt, P. H. 2009, A\&A, 506, 1367

Woitke, P., \& Helling, Ch. 2003, A\&A, 399, 297

Woitke, P., \& Helling, Ch. 2004, A\&A, 414, 335

Wolf, E. T. 2017, ApJL, 839, L1

Zellem, R. T., Griffith, C. A., Pearson, K. A., et al. 2015, ApJ, 810, 11

Zellem, R. T., Swain, M. R., Roudier, G., et al. 2017, ApJ, 844, 27

Zeng, L., Sasselov, D. D., \& Jacobsen, S. B. 2016, ApJ, 819, 127 